\title{
Framework for the chiral extrapolation of the charmed baryon ground-state masses
}

\author{
Yonggoo Heo, ${ }^{1}$ Xiao-Yu Guo, ${ }^{2}$ and Matthias F. M. Lutz ${ }^{2,3}$ \\ ${ }^{1}$ Suranaree University of Technology, Nakhon Ratchasima 30000, Thailand \\ ${ }^{2}$ GSI Helmholtzzentrum für Schwerionenforschung GmbH, Planckstraße 1, 64291 Darmstadt, Germany \\ ${ }^{3}$ Technische Universität Darmstadt, D-64289 Darmstadt, Germany
}

(Received 22 June 2018; published 11 September 2018; corrected 25 October 2018)

\begin{abstract}
We consider the chiral Lagrangian for charmed baryon fields with $J^{P}=\frac{1}{2}^{+}$or $J^{P}=\frac{3}{2}^{+}$quantum numbers. A chiral expansion framework for the baryon ground state masses is worked out to $\mathrm{N}^{3} \mathrm{LO}$ as to compute their dependence on the up, down and strange quark masses for finite box QCD lattice simulations. It is formulated in terms of on-shell meson and baryon masses. The convergence of such a scheme is illustrated with physical masses as taken from the Particle Data Group (PDG). The counter terms relevant at $\mathrm{N}^{3} \mathrm{LO}$ are correlated systematically by large- $N_{c}$ sum rules to leading and subleading order in a manner that keeps the renormalization scale invariance of the approach.
\end{abstract}

DOI: 10.1103/PhysRevD.98.054012

\section{INTRODUCTION}

QCD lattice simulations offer the opportunity to determine low-energy parameters of the chiral Lagrangian. Since the simulations are performed also at quark masses distinct to those needed to reproduce the physical hadron masses new information is generated that may help to determine so far unknown low-energy constants.

Such programs have already been successfully set up for the masses of baryons and mesons in their ground states with $J^{P}=\frac{1}{2}^{+}, \frac{3}{2}^{+}$and $J^{P}=0^{-}, 1^{-}$quantum numbers [1-3]. Corresponding sets of low-energy parameters to be used in flavor SU(3) chiral Lagrangians were established from the available lattice data on such hadron masses $[1,2]$.

The purpose of the present work is to establish a corresponding framework for the masses of charmed baryons, which can then eventually be applied to the current QCD lattice data. Given the rather scarce data set that is provided so far on the charmed baryon masses [4-9] it is important to derive additional constraints from QCD that will guide a fit of the low-energy constants to such data. An important first step in this direction are the recent works $[10,11]$, in which all counterterms that turn relevant in a chiral expansion of the charmed baryon masses are constructed and correlated by the heavyquark spin symmetry and sum rules derived from large$N_{c}$ QCD. Here we complement these results by deriving

Published by the American Physical Society under the terms of the Creative Commons Attribution 4.0 International license. Further distribution of this work must maintain attribution to the author(s) and the published article's title, journal citation, and DOI. Funded by SCOAP. explicit expressions for the various contributions to the baryon masses that arise at next-to-next-to-next-to leading order $\left(\mathrm{N}^{3} \mathrm{LO}\right)$.

As was argued in our previous works [1-3] a chiral expansion around the flavor SU(3) limit of QCD in terms of bare meson and baryon masses is not convergent for the physical up, down and strange quark masses. Any attempt to apply such a conventional expansion strategy to the QCD lattice data set is futile and should be abandoned [12]. Instead, it was demonstrated that a reformulated expansion scheme that uses the on-shell meson and baryon masses appears to have a significantly larger convergence domain, that is applicable to the physical masses for up, down and strange quarks [1,2]. In this work the details of such an approach for the charmed baryon masses are presented. In particular its convergence properties are illustrated at the hand of a chiral decomposition of the bubble loop contributions at physical meson and baryon masses. This is supplemented by the derivation of additional sum rules for the counter terms that arise from the condition of renormalization scale invariance. Given the results of this work an application to the data set from lattice QCD group is feasible. This should eventually lead to a faithful set of low-energy constants.

The work is organized as follows. In Sec. II the relevant parts of the chiral Lagrangian are collected. All expressions required at $\mathrm{N}^{3} \mathrm{LO}$ are detailed in Sec. III. In Sec. IV the various large- $N_{c}$ sum rules are studied at the one-loop level. The paper continues with a convergence study in Secs. V and VI of the one-loop bubble contributions as decomposed into their chiral moments. A short summary given with Sec. VII. The Appendix provides a glossary for our notations and conventions. 


\section{CHIRAL LAGRANGIAN WITH CHARMED BARYON FIELDS}

The chiral Lagrangian is a reliable tool, once it is combined with appropriate counting rules leading to a systematic approximation strategy. In the following we recall the leading order (LO) terms $[10,13,14]$. It is convenient to decompose the fields into their isospin multiplets with

$$
\begin{aligned}
\Phi & =\tau \cdot \pi(140)+\alpha^{\dagger} \cdot K(494)+K^{\dagger}(494) \cdot \alpha+\eta(547) \lambda_{8}, \\
\sqrt{2} B_{[\overline{3}]} & =\frac{1}{\sqrt{2}} \alpha^{\dagger} \cdot \Xi_{c}(2470)-\frac{1}{\sqrt{2}} \Xi_{c}^{T}(2470) \cdot \alpha+i \tau_{2} \Lambda_{c}(2284), \\
\sqrt{2} B_{[6]} & =\frac{1}{\sqrt{2}} \alpha^{\dagger} \cdot \Xi_{c}^{\prime}(2580)+\frac{1}{\sqrt{2}} \Xi_{c}^{\prime T}(2580) \cdot \alpha+\Sigma_{c}(2455) \cdot \tau i \tau_{2}+\frac{\sqrt{2}}{3}\left(1-\sqrt{3} \lambda_{8}\right) \Omega_{c}(2704), \\
\sqrt{2} B_{[6]}^{\mu} & =\frac{1}{\sqrt{2}} \alpha^{\dagger} \cdot \Xi_{c}^{\mu}(2645)+\frac{1}{\sqrt{2}} \Xi_{c}^{T, \mu}(2645) \cdot \alpha+\Sigma_{c}^{\mu}(2520) \cdot \tau i \tau_{2}+\frac{\sqrt{2}}{3}\left(1-\sqrt{3} \lambda_{8}\right) \Omega_{c}^{\mu}(2770), \\
\alpha^{\dagger} & =\frac{1}{\sqrt{2}}\left(\lambda_{4}+i \lambda_{5}, \lambda_{6}+i \lambda_{7}\right), \\
\tau & =\left(\lambda_{1}, \lambda_{2}, \lambda_{3}\right),
\end{aligned}
$$

where the matrices $\lambda_{i}$ are the standard Gell-Mann generators of the $\mathrm{SU}(3)$ algebra. The numbers in the brackets recall the approximate masses of the particles in units of $\mathrm{MeV}$. Note that we do not consider the $\eta^{\prime}$ meson as an active degree of freedom in our current study $[15,16]$.

It should be noted that $\Xi_{c}$ and $\Xi_{c}^{\prime}$ have the same quantum numbers and therefore a mixing of the two fields needs to be considered [17]. We introduce a $\Xi_{c}-\Xi_{c}^{\prime}$ mixing angle $\epsilon$ by

$$
\begin{gathered}
\Xi_{c}=\bar{\Xi}_{c} \cos \epsilon+\bar{\Xi}_{c}^{\prime} \sin \epsilon, \quad \Xi_{c}^{\prime}=\bar{\Xi}_{c}^{\prime} \cos \epsilon-\bar{\Xi}_{c} \sin \epsilon, \\
\text { with } \quad \Sigma_{\Xi_{c} \Xi_{c}^{\prime}}=\frac{1}{2}\left(\Sigma_{\Xi_{c}^{\prime}}-\Sigma_{\Xi_{c}}\right) \tan (2 \epsilon),
\end{gathered}
$$

where the physical fields are denoted by $\bar{\Xi}_{c}$ and $\bar{\Xi}_{c}^{\prime}$. The offdiagonal self-energy $\Sigma_{\Xi_{c} \Xi_{c}^{\prime}} \neq 0$ reflects the fact that the fields $\Xi_{c}$ and $\Xi_{c}^{\prime}$ are unphysical. Only for the physical fields $\bar{\Xi}_{c}$ and $\bar{\Xi}_{c}^{\prime}$ we expect their corresponding off-diagonal selfenergy $\Sigma_{\bar{\Xi}_{c} \bar{\Xi}_{c}^{\prime}} \rightarrow 0$ to vanish for on-shell conditions.

There are the kinetic terms

$$
\begin{aligned}
\mathcal{L}^{(1)}= & \operatorname{tr} \bar{B}_{[6]}\left(\gamma^{\mu} i D_{\mu}-M_{[6]}^{1 / 2}\right) B_{[6]}-\operatorname{tr}\left(\overline { B } _ { [ 6 ] } ^ { \mu } \left(\left[i \not D-M_{[6]}^{3 / 2}\right] g_{\mu \nu}-i\left(\gamma_{\mu} D_{\nu}+\gamma_{\nu} D_{\mu}\right)\right.\right. \\
& \left.\left.+\gamma_{\mu}\left[i \not D+M_{[6]}^{3 / 2}\right] \gamma_{\nu}\right) B_{[6]}^{\nu}\right)+\operatorname{tr} \bar{B}_{[\overline{3}]}\left(\gamma^{\mu} i D_{\mu}-M_{[\overline{3}]}^{1 / 2}\right) B_{[\overline{3}]}+F_{[66]} \operatorname{tr} \bar{B}_{[6]} \gamma^{\mu} \gamma_{5} i U_{\mu} B_{[6]}+F_{[\overline{3} \overline{3}]} \operatorname{tr} \bar{B}_{[\overline{3}]} \gamma^{\mu} \gamma_{5} i U_{\mu} B_{[\overline{3}]} \\
& +F_{[\overline{3} 6]} \operatorname{tr}\left(\bar{B}_{[6]} \gamma^{\mu} \gamma_{5} i U_{\mu} B_{[\overline{3}]}+\text { H.c. }\right)+C_{[66]} \operatorname{tr}\left(\bar{B}_{[6]}^{\mu} i U_{\mu} B_{[6]}+\text { H.c. }\right)+C_{[\overline{3} 6]} \operatorname{tr}\left(\bar{B}_{[6]}^{\mu} i U_{\mu} B_{[\overline{3}]}+\text { H.c. }\right) \\
& -H_{[66]} \operatorname{tr} \bar{B}_{[6]}^{\alpha} g_{\alpha \beta} \gamma^{\mu} \gamma_{5} i U_{\mu} B_{[6]}^{\beta}, \\
U_{\mu}= & \frac{1}{2} u^{\dagger}\left(\partial_{\mu} e^{i \frac{\Phi}{f}}\right) u^{\dagger}-\frac{i}{2} u^{\dagger}\left(v_{\mu}+a_{\mu}\right) u+\frac{i}{2} u\left(v_{\mu}-a_{\mu}\right) u^{\dagger}, \quad u=e^{i \frac{\Phi}{2 f f}}, \\
D_{\mu} B= & \partial_{\mu} B+\Gamma_{\mu} B+B \Gamma_{\mu}^{T}, \\
\Gamma_{\mu}= & \frac{1}{2} u^{\dagger}\left[\partial_{\mu}-i\left(v_{\mu}+a_{\mu}\right)\right] u+\frac{1}{2} u\left[\partial_{\mu}-i\left(v_{\mu}-a_{\mu}\right)\right] u^{\dagger},
\end{aligned}
$$

and 6 structures which parametrize the three-point interactions of the Goldstone bosons with the charmed baryon fields $[13,14]$. From the kinetic terms one can read off the Weinberg-Tomozawa interaction terms on which the coupled-channel computation of [18] rests. It follows upon an expansion of the kinetic terms in powers of the Goldstone boson fields. At leading order in a chiral expansion, the bare masses $M_{[6]}^{1 / 2}, M_{[6]}^{3 / 2}$ and $M_{[\overline{3}]}^{1 / 2}$ may be identified with the flavor average of the sextet and antitriplet baryon masses. Note the classical vector and axial-vector source functions $v_{\mu}$ and $a_{\mu}$ of QCD in (3) were instrumental in the derivation of our large- $N_{c}$ sum rules $[10,11]$. 
We proceed with the terms at next-to-leading order (NLO) where there are symmetry conserving and symmetry breaking terms $[10,11]$. We recall the 7 symmetry breaking counter terms

$$
\begin{aligned}
\mathcal{L}_{\chi}^{(2)}= & b_{1,[\overline{3} \overline{3}]} \operatorname{tr}\left(\bar{B}_{[\overline{3}]} B_{[\overline{3}]}\right) \operatorname{tr}\left(\chi_{+}\right)+b_{2,[\overline{3} \overline{3}]} \operatorname{tr}\left(\bar{B}_{[\overline{3}]} \chi_{+} B_{[\overline{[}]}\right)+b_{1,[\overline{3} 6]} \operatorname{tr}\left(\bar{B}_{[6]} \chi_{+} B_{[\overline{3}]}+\text { H.c. }\right) \\
& +b_{1,[66]} \operatorname{tr}\left(\bar{B}_{[6]} B_{[6]}\right) \operatorname{tr}\left(\chi_{+}\right)+b_{2,[66]} \operatorname{tr}\left(\bar{B}_{[6]} \chi_{+} B_{[6]}\right)-d_{1,[66]} \operatorname{tr}\left(g_{\mu \nu} \bar{B}_{[6]}^{\mu} B_{[6]}^{\nu}\right) \operatorname{tr}\left(\chi_{+}\right)-d_{2,[66]} \operatorname{tr}\left(g_{\mu \nu} \bar{B}_{[6]}^{\mu} \chi_{+} B_{[6]}^{\nu}\right), \\
\chi_{+}= & \frac{1}{2}\left(u \chi_{0} u+u^{\dagger} \chi_{0} u^{\dagger}\right),
\end{aligned}
$$

with $\chi_{0}=2 B_{0} \operatorname{diag}\left(m, m, m_{s}\right)$ proportional to the quark-mass matrix. We do not consider isospin violating effects in this work. The low-energy constants of (4) imply a linear quark-mass dependence for the charmed baryon masses with

$$
\begin{aligned}
M_{\Xi_{c}}^{(2)}-M_{\Lambda_{c}}^{(2)} & =-B_{0}\left(m_{s}-m\right) b_{2,[\overline{3} \overline{3}]}, \\
M_{\Xi_{c}^{\prime}}^{(2)}-M_{\Sigma_{c}}^{(2)} & =-B_{0}\left(m_{s}-m\right) b_{2,[66]}, \quad M_{\Omega_{c}}^{(2)}-M_{\Sigma_{c}}^{(2)}=-2 B_{0}\left(m_{s}-m\right) b_{2,[66]}, \\
M_{\Xi_{c}^{*}}^{(2)}-M_{\Sigma_{c}^{*}}^{(2)} & =-B_{0}\left(m_{s}-m\right) d_{2,[66]}, \quad M_{\Omega_{c}^{*}}^{(2)}-M_{\Sigma_{c}^{*}}^{(2)}=-2 B_{0}\left(m_{s}-m\right) d_{2,[66]}, \\
M_{\Lambda_{c}}^{(2)}+2 M_{\Xi_{c}}^{(2)} & =-2 B_{0}\left(2 m+m_{s}\right)\left(3 b_{1,[\overline{3} \overline{3}]}+b_{2,[\overline{3} \overline{3}]}\right), \\
M_{\Omega_{c}}^{(2)}+2 M_{\Xi_{c}^{\prime}}^{(2)}+3 M_{\Sigma_{c}}^{(2)} & =-4 B_{0}\left(2 m+m_{s}\right)\left(3 b_{1,[66]}+b_{2,[66]}\right), \\
M_{\Omega_{c}^{*}}^{(2)}+2 M_{\Xi_{c}^{*}}^{(2)}+3 M_{\Sigma_{c}^{*}}^{(2)} & =-4 B_{0}\left(2 m+m_{s}\right)\left(3 d_{1,[66]}+d_{2,[66]}\right), \\
M_{\Xi_{c} \Xi_{c}^{\prime}}^{(2)} & =B_{0}\left(m_{s}-m\right) b_{1,[\overline{3} 6]},
\end{aligned}
$$

where the upper index 2 in $M_{B}^{(2)}$ projects the mass of the baryon of type $B$ on its chiral order $Q^{2}$ in this case.

A complete list of chiral symmetry conserving $Q^{2}$ counterterms, relevant for the calculation of the charm baryon masses at $\mathrm{N}^{3} \mathrm{LO}$, was given in $[10,11]$. In these works the $Q^{2}$ counter terms are grouped according to their Dirac structure. Here we display the scalar and vector terms relevant for our study only

$$
\mathcal{L}^{(2)}=\mathcal{L}^{(S)}+\mathcal{L}^{(V)},
$$

with

$$
\begin{aligned}
& \mathcal{L}^{(S)}=-g_{0,[\overline{3} \overline{3}]}^{(S)} \operatorname{tr}\left(\bar{B}_{[\overline{3}]} B_{[\overline{3}]}\right) \operatorname{tr}\left(U_{\mu} U^{\mu}\right)-g_{D,[\overline{3} \overline{3}]}^{(S)} \operatorname{tr}\left(\bar{B}_{[\overline{3}]}\left\{U_{\mu}, U^{\mu}\right\} B_{[\overline{3}]}\right)-g_{0,[66]}^{(S)} \operatorname{tr}\left(\bar{B}_{[6]} B_{[6]}\right) \operatorname{tr}\left(U_{\mu} U^{\mu}\right)-g_{1,[66]}^{(S)} \operatorname{tr}\left(\bar{B}_{[6]} U^{\mu} B_{[6]} U_{\mu}^{T}\right) \\
& -g_{D,[66]}^{(S)} \operatorname{tr}\left(\bar{B}_{[6]}\left\{U_{\mu}, U^{\mu}\right\} B_{[6]}\right)-g_{D,[\overline{3} 6]}^{(S)} \operatorname{tr}\left(\bar{B}_{[6]}\left\{U_{\mu}, U^{\mu}\right\} B_{[\overline{3}]}+\text { H.c. }\right) \\
& +h_{0,[66]}^{(S)} \operatorname{tr}\left(\bar{B}_{[6]}^{\mu} g_{\mu \nu} B_{[6]}^{\nu}\right) \operatorname{tr}\left(U_{\alpha} U^{\alpha}\right)+h_{1,[66]}^{(S)} \operatorname{tr}\left(\bar{B}_{[6]}^{\mu} B_{[6]}^{\nu}\right) \operatorname{tr}\left(U_{\mu} U_{\nu}\right)+h_{2,[66]}^{(S)} \operatorname{tr}\left(\bar{B}_{[6]}^{\mu} g_{\mu \nu}\left\{U^{\alpha}, U_{\alpha}\right\} B_{[6]}^{\nu}\right) \\
& -h_{3,[66]}^{(S)} \operatorname{tr}\left(\bar{B}_{[6]}^{\mu}\left\{U_{\mu}, U_{\nu}\right\} B_{[6]}^{\nu}\right)+h_{4,[66]}^{(S)} \operatorname{tr}\left(\bar{B}_{[6]}^{\mu} g_{\mu \nu} U^{\alpha} B_{[6]}^{\nu} U_{\alpha}^{T}\right)+h_{5,[66]}^{(S)} \operatorname{tr}\left(\bar{B}_{[6]}^{\mu} U_{\nu} B_{[6]}^{\nu} U_{\mu}^{T}+\bar{B}_{[6]}^{\mu} U_{\mu} B_{[6]}^{\nu} U_{\nu}^{T}\right), \\
& \mathcal{L}^{(V)}=-\frac{1}{2} g_{0,[\overline{3} \overline{3}]}^{(V)} \operatorname{tr}\left(\bar{B}_{[\overline{[}]} i \gamma^{\alpha}\left(D^{\beta} B_{[\overline{3}]}\right) \operatorname{tr}\left(U_{\beta} U_{\alpha}\right)+\text { H.c. }\right) \\
& -\frac{1}{2} g_{1,[\overline{3} \overline{3}]}^{(V)} \operatorname{tr}\left(\bar{B}_{[\overline{3}]} i \gamma^{\alpha} U_{\beta}\left(D^{\beta} B_{[\overline{3}]}\right) U_{\alpha}^{T}+\bar{B}_{[\overline{3}]} i \gamma^{\alpha} U_{\alpha}\left(D^{\beta} B_{[\overline{3}]}\right) U_{\beta}^{T}+\text { H.c. }\right)-\frac{1}{2} g_{D,[\overline{3} \overline{3}]}^{(V)} \operatorname{tr}\left(\bar{B}_{[\overline{3}]} i \gamma^{\alpha}\left\{U_{\alpha}, U_{\beta}\right\}\left(D^{\beta} B_{[\overline{3}]}\right)+\text { H.c. }\right) \\
& -\frac{1}{2} g_{D,[\overline{3} 6]}^{(V)} \operatorname{tr}\left(\bar{B}_{[6]} i \gamma^{\alpha}\left\{U_{\alpha}, U_{\beta}\right\}\left(D^{\beta} B_{[\overline{3}]}\right)-\left(D^{\beta} \bar{B}_{[6]}\right) i \gamma^{\alpha}\left\{U_{\alpha}, U_{\beta}\right\} B_{[\overline{3}]}+\text { H.c. }\right)-\frac{1}{2} g_{0,[66]}^{(V)}\left(\operatorname{tr}\left(\bar{B}_{[6]} i \gamma^{\alpha}\left(D^{\beta} B_{[6]}\right)\right) \operatorname{tr}\left(U_{\beta} U_{\alpha}\right)+\text { H.c. }\right) \\
& -\frac{1}{4} g_{1,[6]}^{(V)} \operatorname{tr}\left(\bar{B}_{[6]} i \gamma^{\alpha} U_{\beta}\left(D^{\beta} B_{[6]}\right) U_{\alpha}^{T}+\bar{B}_{[6]} i \gamma^{\alpha} U_{\alpha}\left(D^{\beta} B_{[6]}\right) U_{\beta}^{T}+\text { H.c. }\right)-\frac{1}{2} g_{D,[66]}^{(V)} \operatorname{tr}\left(\bar{B}_{[6]} i \gamma^{\alpha}\left\{U_{\alpha}, U_{\beta}\right\}\left(D^{\beta} B_{[6]}\right)+\text { H.c. }\right) \\
& +\frac{1}{2} h_{0,[66]}^{(V)} \operatorname{tr}\left(\bar{B}_{[6]}^{\mu} g_{\mu \nu} i \gamma^{\alpha}\left(D^{\beta} B_{[6]}^{\nu}\right) \operatorname{tr}\left(U_{\alpha} U_{\beta}\right)+\text { H.c. }\right)+\frac{1}{4} h_{1,[66]}^{(V)} \operatorname{tr}\left(\bar{B}_{[6]}^{\mu} g_{\mu \nu} i \gamma^{\alpha} U_{\beta}\left(D^{\beta} B_{[6]}^{\nu}\right) U_{\alpha}^{T}+\bar{B}_{[6]}^{\mu} g_{\mu \nu} i \gamma^{\alpha} U_{\alpha}\left(D^{\beta} B_{[6]}^{\nu}\right) U_{\beta}^{T}+\text { H.c. }\right) \\
& +\frac{1}{2} h_{2,[66]}^{(V)} \operatorname{tr}\left(\bar{B}_{[6]}^{\mu} g_{\mu \nu} i \gamma^{\alpha}\left\{U_{\alpha}, U_{\beta}\right\}\left(D^{\beta} B_{[6]}^{\nu}\right)+\text { H.c. }\right) \text {, }
\end{aligned}
$$


where further possible terms that are redundant owing to the on-shell conditions of spin- $\frac{3}{2}$ fields with $\gamma_{\mu} B_{[6]}^{\mu}=0$ and $\partial_{\mu} B_{[6]}^{\mu}=0$ are eliminated systematically.

The counterterms recalled in (7) contribute to the baryon masses at the one-loop level. They imply renormalization scale dependent contributions that need to be balanced by a set of symmetry breaking counterterms in $\mathcal{L}_{\chi}^{(4)}$. We close this section with a partial collection of terms contributing to $\mathcal{L}_{\chi}^{(4)}$ that are relevant in a chiral extrapolation of the baryon masses at $\mathrm{N}^{3} \mathrm{LO}$. There are 16 such symmetry breaking counter terms

$$
\begin{aligned}
\mathcal{L}_{\chi}^{(4)}= & c_{1,[\overline{3} \overline{3}]} \operatorname{tr}\left(\bar{B}_{[\overline{3}]} B_{[\overline{3}]}\right) \operatorname{tr}\left(\chi_{+}^{2}\right)+c_{2,[\overline{3} \overline{3}]} \operatorname{tr}\left(\bar{B}_{[\overline{3}]} B_{[\overline{3}]}\right)\left(\operatorname{tr} \chi_{+}\right)^{2}+c_{3,[\overline{3} \overline{3}]} \operatorname{tr}\left(\bar{B}_{[\overline{3}]} \chi_{+} B_{[\overline{3}]}\right) \operatorname{tr}\left(\chi_{+}\right)+c_{4,[\overline{3} \overline{3}]} \operatorname{tr}\left(\bar{B}_{[\overline{3}]} \chi_{+}^{2} B_{[\overline{3}]}\right) \\
& +c_{1,[66]} \operatorname{tr}\left(\bar{B}_{[6]} B_{[6]}\right) \operatorname{tr}\left(\chi_{+}^{2}\right)+c_{2,[66]} \operatorname{tr}\left(\bar{B}_{[6]} B_{[6]}\right)\left(\operatorname{tr} \chi_{+}\right)^{2}+c_{3,[66]} \operatorname{tr}\left(\bar{B}_{[6]} \chi_{+} B_{[6]}\right) \operatorname{tr}\left(\chi_{+}\right)+c_{4,[66]} \operatorname{tr}\left(\bar{B}_{[6]} \chi_{+}^{2} B_{[6]}\right) \\
& +c_{5,[66]} \operatorname{tr}\left(\bar{B}_{[6]} \chi_{+} B_{[6]} \chi_{+}^{T}\right)+c_{1,[\overline{3} 6]} \operatorname{tr}\left(\bar{B}_{[6]} \chi_{+} B_{[\overline{3}]}+\text { H.c. }\right) \operatorname{tr}\left(\chi_{+}\right)+c_{2,[\overline{3} 6]} \operatorname{tr}\left(\bar{B}_{[6]} \chi_{+}^{2} B_{[\overline{3}]}+\text { H.c. }\right) \\
& -e_{1,[66]} \operatorname{tr}\left(\bar{B}_{[6]}^{\mu} g_{\mu \nu} B_{[6]}^{\nu}\right) \operatorname{tr}\left(\chi_{+}^{2}\right)-e_{2,[66]} \operatorname{tr}\left(\bar{B}_{[6]}^{\mu} g_{\mu \nu} B_{[6]}^{\nu}\right)\left(\operatorname{tr} \chi_{+}\right)^{2}-e_{3,[66]} \operatorname{tr}\left(\bar{B}_{[6]}^{\mu} g_{\mu \nu} \chi_{+} B_{[6]}^{\nu}\right) \operatorname{tr}\left(\chi_{+}\right) \\
& -e_{4,[66]} \operatorname{tr}\left(\bar{B}_{[6]}^{\mu} g_{\mu \nu} \chi_{+}^{2} B_{[6]}^{\nu}\right)-e_{5,[66]} \operatorname{tr}\left(\bar{B}_{[6]}^{\mu} g_{\mu \nu} \chi_{+} B_{[6]}^{\nu} \chi_{+}^{T}\right) .
\end{aligned}
$$

Altogether we count 54 low-energy constants in this section that have to be determined by some data set. Clearly, any additional constraints from heavy-quark spin symmetry or large- $N_{c}$ QCD are desperately needed to arrive at any significant result. Such constraints were derived in $[10,11,19]$ to subleading order in the $1 / N_{c}$ expansion and are summarized in Appendix A for the readers' convenience.

\section{CHIRAL EXPANSION OF THE CHARMED BARYON MASSES}

We turn to the computation of the baryon masses. The baryon self-energy, $\Sigma_{B}(\not p)$, may be considered to be a function of $p_{\mu} \gamma^{\mu}$ only, with the 4-momentum $p_{\mu}$ of the baryon $B$. This is obvious for the spin-one-half baryons, but less immediate for the spin-three-half baryons. We refer to [20] for technical details. To order $Q^{4}$ the self-energy receives contributions of symmetry breaking counterterms, the tadpole and the one-loop bubble diagram

$$
\Sigma_{B}\left(M_{B}\right)=\Sigma_{B}^{\text {tree-level }}+\Sigma_{B}^{\text {tadpole }}+\Sigma_{B}^{\text {bubble }}
$$

where the index $B$ stands for the members of the flavor multiplets with $J^{P}=\frac{1}{2}^{+}, \frac{3}{2}{ }^{+}$. Since the $J^{P}=\frac{1}{2}^{+}$states come either in a flavor antitriplet or a flavor sextet we discriminate those states by $B \in[\overline{3}]$ and $B \in[6]$. In contrast, the $J^{P}=\frac{3}{2}^{+}$ground states are realized only in a flavor sextet. In order to keep these states apart from the sextet with $J^{P}=\frac{1}{2}^{+}$we label the $J^{P}=\frac{3}{2}{ }^{+}$states by $B \in[4]$ where we refer to the spin rather than the flavor multiplicity in this case.

The on-shell mass of the baryon $M_{B}$ is determined by the condition

$$
M_{B}-\Sigma_{B}\left(M_{B}\right)=M_{B}^{(0)}= \begin{cases}\bar{M}_{[\overline{[}]}^{J=1 / 2} \equiv M_{[\overline{3}]} & \text { for } B \in[\overline{3}] \\ \bar{M}_{[6]}^{J=1 / 2} \equiv M_{[6]} \text { for } B \in[6] \\ \bar{M}_{[6]}^{J=3 / 2} \equiv M_{[4]} \text { for } B \in[4]\end{cases}
$$

where $M_{[\overline{3}}, M_{[4]}$ and $M_{[6]}$ are the renormalized and scaleindependent masses of the baryon multiplets in the flavor $S U(3)$ limit.

The separation of the baryon self-energies into a loop and a tree-level contribution is not unique depending on the renormalization scheme. In this work we apply a recent approach developed for the chiral extrapolation of the baryon octet and decuplet masses [1,2,21]. It is based on the $\chi$ MS scheme [20] and can directly be adapted to the charmed baryons, the focus of the current work. A matching with alternative renormalization schemes is most economically performed by a direct comparison with the explicit expressions of our study. Given the renormalization scheme [1] the low-energy constants $b_{n}$ or $d_{n}$ do specify the linear quark mass dependence of the baryon masses as already detailed in (5). The particular subtraction scheme for the loop contributions as introduced in [1] was constructed to ensure this property of $b_{n}$ or $d_{n}$.

Let us begin with the tadpole contributions, which in a finite volume take the following form

$$
\Sigma_{B}^{\mathrm{tadpole}}=\frac{1}{(2 f)^{2}} \sum_{Q \in[8]}\left(G_{B Q}^{(\chi)} \bar{I}_{Q}^{(0)}-m_{Q}^{2} G_{B Q}^{(S)} \bar{I}_{Q}^{(0)}-M_{B}^{(0)} G_{B Q}^{(V)} \bar{I}_{Q}^{(2)}\right),
$$

where the various Clebsch coefficients $G_{B Q}^{(\chi)}$ and $G_{B Q}^{(S)}, G_{B Q}^{(V)}$ are summarized in Table I for the $J^{P}=\frac{1}{2}^{+}$states. ${ }^{1}$ Note that

\footnotetext{
${ }^{1}$ For the tadpole contribution to the $\Xi_{c} \Xi_{c}^{\prime}$ mixing it follows $M_{B}^{(0)}=\frac{1}{2}\left(M_{\overline{3}]}+M_{[6]}\right)$.
} 
TABLE I. The Clebsch coefficients $G_{Q B}^{(\chi)}$ and $G_{Q B}^{(V)}$ of $(11)$ for the $J^{P}=\frac{1}{2}^{+}$states. We assure that the scalar $G_{Q B}^{(S)}$ follow from the vector

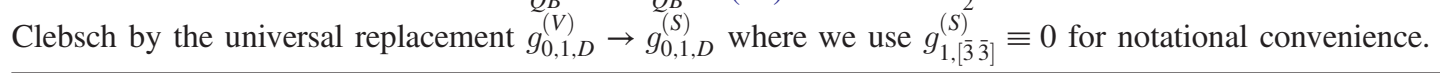

\begin{tabular}{|c|c|c|c|}
\hline$B$ & $Q$ & $G_{B Q}^{(\chi)}$ & $G_{B Q}^{(V)}$ \\
\hline \multirow[t]{3}{*}{$\Lambda_{c}$} & $\pi$ & $12 B_{0}\left(2 b_{1,[\overline{3} \overline{3}]}+b_{2,[\overline{3} \overline{3}]}\right) m$ & $6\left(g_{0,[\overline{3} \overline{3}]}^{(V)}-g_{1,[\overline{3} \overline{3}]}^{(V)}+g_{D,[\overline{3} \overline{3}]}^{(V)}\right)$ \\
\hline & $K$ & $4 B_{0}\left(4 b_{1,[\overline{3} \overline{3}]}+b_{2,[\overline{3} \overline{3}]}\right)\left(m+m_{s}\right)$ & $8 g_{0,[\overline{3} \overline{3}]}^{(V)}+4 g_{D,[\overline{3} \overline{3}]}^{(V)}$ \\
\hline & $\eta$ & $\frac{4}{3} B_{0}\left(b_{2,[\overline{3} \overline{3}]} m+2 b_{1,[\overline{3} \overline{3}]}\left(m+2 m_{s}\right)\right)$ & $2 g_{0,[\overline{3} \overline{3}]}^{(V)}+\frac{2}{3} g_{1,[\overline{3} \overline{3}]}^{(V)}+\frac{2}{3} g_{D,[\overline{3} \overline{3}]}^{(V)}$ \\
\hline \multirow[t]{3}{*}{$\Xi_{c}$} & $\pi$ & $6 B_{0}\left(4 b_{1,[\overline{3} \overline{3}]}+b_{2,[\overline{3} \overline{3}]}\right) m$ & $6 g_{0,[\overline{3} \overline{3}]}^{(V)}+3 g_{D,[\overline{3} \overline{3}]}^{(V)}$ \\
\hline & $K$ & $2 B_{0}\left(8 b_{1,[\overline{3} \overline{3}]}+3 b_{2,[\overline{3} \overline{3}]}\right)\left(m+m_{s}\right)$ & $8 g_{0,[\overline{3} \overline{3}]}^{(V)}-4 g_{1,[\overline{3} \overline{3}]}^{(V)}+6 g_{D,[\overline{3} \overline{3}]}^{(V)}$ \\
\hline & $\eta$ & $\frac{2}{3} B_{0}\left(4 b_{1,[\overline{3} \overline{3}]}\left(m+2 m_{s}\right)+b_{2,[\overline{3} \overline{3}]}\left(m+4 m_{s}\right)\right)$ & $2 g_{0,[\overline{3} \overline{3}]}^{(V)}-\frac{4}{3} g_{1,[\overline{3} \overline{3}]}^{(V)}+\frac{5}{3} g_{D,[\overline{3} \overline{3}]}^{(V)}$ \\
\hline \multirow[t]{3}{*}{$\Xi_{c} \Xi_{c}^{\prime}$} & $\pi$ & $6 B_{0} b_{1,[\overline{3} 6]} m$ & $3 g_{D,[\overline{3} 6]}^{(V)}$ \\
\hline & $K$ & $-2 B_{0} b_{1,[\overline{3} 6]}\left(m+m_{s}\right)$ & $-2 g_{D,[\overline{3} 6]}^{(V)}$ \\
\hline & $\eta$ & $-\frac{2}{3} B_{0} b_{1,[\overline{3} 6]}\left(4 m_{s}-m\right)$ & $-g_{D,[\overline{3} 6]}^{(V)}$ \\
\hline \multirow[t]{3}{*}{$\Sigma_{c}$} & $\pi$ & $12 B_{0}\left(2 b_{1,[66]}+b_{2,[66]}\right) m$ & $6 g_{0,[66]}^{(V)}+g_{1,[66]}^{(V)}+6 g_{D,[66]}^{(V)}$ \\
\hline & $K$ & $4 B_{0}\left(4 b_{1,[66]}+b_{2,[66]}\right)\left(m+m_{s}\right)$ & $8 g_{0,[66]}^{(V)}+4 g_{D,[66]}^{(V)}$ \\
\hline & $\eta$ & $\frac{4}{3} B_{0}\left(b_{2,[66]} m+2 b_{1,[66]}\left(m+2 m_{s}\right)\right)$ & $2 g_{0,[66]}^{(V)}+\frac{1}{3} g_{1,[66]}^{(V)}+\frac{2}{3} g_{D,[66]}^{(V)}$ \\
\hline \multirow[t]{3}{*}{$\Xi_{c}^{\prime}$} & $\pi$ & $6 B_{0}\left(4 b_{1,[66]}+b_{2,[66]}\right) m$ & $6 g_{0,[66]}^{(V)}+3 g_{D,[66]}^{(V)}$ \\
\hline & $K$ & $2 B_{0}\left(8 b_{1,[66]}+3 b_{2,[66]}\right)\left(m+m_{s}\right)$ & $8 g_{0,[66]}^{(V)}+2 g_{1,[66]}^{(V)}+6 g_{D,[66]}^{(V)}$ \\
\hline & $\eta$ & $\frac{2}{3} B_{0}\left(4 b_{1,[66]}\left(m+2 m_{s}\right)+b_{2,[66]}\left(m+4 m_{s}\right)\right)$ & $2 g_{0,[66]}^{(V)}-\frac{2}{3} g_{1,[66]}^{(V)}+\frac{5}{3} g_{D,[66]}^{(V)}$ \\
\hline \multirow[t]{3}{*}{$\Omega_{c}$} & $\pi$ & $24 B_{0} b_{1,[66]} m$ & $6 g_{0,[66]}^{(V)}$ \\
\hline & $K$ & $8 B_{0}\left(2 b_{1,[66]}+b_{2,[66]}\right)\left(m+m_{s}\right)$ & $8\left(g_{0,[66]}^{(V)}+g_{D,[66]}^{(V)}\right)$ \\
\hline & $\eta$ & $\frac{8}{3} B_{0}\left(b_{1,[66]} m+2\left(b_{1,[66]}+b_{2,[66]}\right) m_{s}\right)$ & $2 g_{0,[66]}^{(V)}+\frac{4}{3} g_{1,[66]}^{(V)}+\frac{8}{3} g_{D,[66]}^{(V)}$ \\
\hline
\end{tabular}

like in our previous works [1-3] we use the letter $Q$ in a context specific manner. It may either denote a chiral order, or as in (11) or Table I if used as an index it runs over the eight Goldstone bosons properly grouped into their isospin multiplets. The set of finite-box scalar tadpole integrals $\bar{I}_{Q}^{(n)}$ were introduced in [21]. Here we recall their infinite volume limit only,

$$
\bar{I}_{Q}^{(0)} \rightarrow \frac{m_{Q}^{2}}{(4 \pi)^{2}} \ln \left(\frac{m_{Q}^{2}}{\mu^{2}}\right), \quad \bar{I}_{Q}^{(2)} \rightarrow \frac{1}{4} m_{Q}^{2} \bar{I}_{Q}^{(0)}
$$

with the renormalization scale $\mu$ of dimensional regularization. Explicit expressions for $\bar{I}_{Q}^{(0)}$ and $\bar{I}_{Q}^{(2)}$ appropriate for their finite volume generalization are given in Eqs. (2) and (19) of [21].

It remains to detail the Clebsch coefficients $G_{Q B}^{(\chi)}$ and $G_{Q B}^{(S, V)}$ for the $J^{P}=\frac{3}{2}+$ states in the flavor sextet states. To do so it is useful to introduce the particular combinations

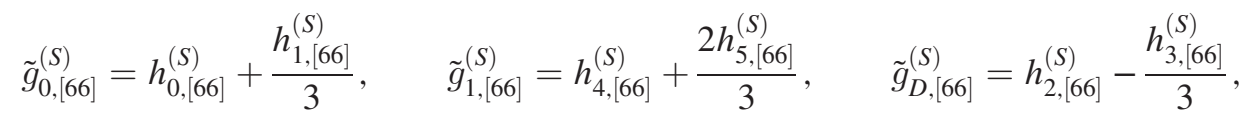

$$
\begin{aligned}
& \tilde{g}_{0,[66]}^{(V)}=h_{0,[66]}^{(V)}-\frac{h_{1,[66]}^{(S)}}{3 M_{[4]}}, \quad \tilde{g}_{1,[66]}^{(V)}=h_{1,[66]}^{(V)}-\frac{2 h_{5,[66]}^{(S)}}{3 M_{[4]}}, \quad \tilde{g}_{D,[66]}^{(V)}=h_{2,[66]}^{(V)}+\frac{h_{3,[66]}^{(S)}}{3 M_{[4]}},
\end{aligned}
$$

such that the desired Clebsch coefficients can be read off from Table I by replacing $g_{0,1, D}^{(S, V)} \rightarrow \tilde{g}_{0,1, D}^{(S, V)}$ together with $b_{x} \rightarrow d_{x}$. 
Consider now the terms quadratic in the quark masses, which we denote with $\Sigma_{B}^{(4-\chi)}$ and are supposed to absorb the renormalization scale dependence of the tadpole terms $\Sigma_{B}^{\text {tadpole }}$. They supplement the terms linear in the quark masses, which were already considered in (5). Together,

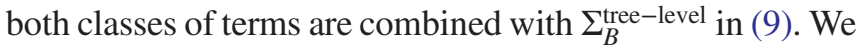

follow here our previous works $[1,2,21]$ in which we keep the on-shell meson masses in the tadpole contributions. This requires us to cast the terms quadratic in the quark masses into corresponding terms that depend on the meson masses in addition. For that purpose we introduce particular parameter combinations

$$
\begin{aligned}
& \tilde{c}_{1,[\overline{3} \overline{3}]}=-\frac{4}{33}\left(33 c_{1,[\overline{3} \overline{3}]}-45 c_{2,[\overline{3} \overline{3}]}-15 c_{3,[\overline{3} \overline{3}]}+11 c_{4,[\overline{3} \overline{3}]}\right), \\
& \tilde{c}_{2,[\overline{3} \overline{3}]}=-\frac{2}{253}\left(207 c_{2,[\overline{3} \overline{3}]}+3 c_{3,[\overline{3} \overline{3}]}-22 c_{4,[\overline{3} \overline{3}]}\right), \\
& \tilde{c}_{3,[\overline{3} \overline{3}]}=-\frac{1}{46}\left(9 c_{3,[\overline{3} \overline{3}]}+26 c_{4,[\overline{3} \overline{3}]}\right), \quad \tilde{c}_{4,[\overline{3} \overline{3}]}=-\frac{1}{92}\left(3 c_{3,[\overline{3} \overline{3}]}-22 c_{4,[\overline{3} \overline{3}]}\right), \\
& \tilde{c}_{1,[\overline{3} 6]}=\frac{1}{46}\left(9 c_{1,[\overline{3} 6]}+26 c_{2,[\overline{3} 6]}\right), \quad \tilde{c}_{2,[\overline{3} 6]}=\frac{3}{92}\left(3 c_{1,[\overline{3} 6]}-22 c_{2,[\overline{3} 6]}\right),
\end{aligned}
$$

in terms of which our unambiguous results are simplified significantly. The self-energies for the flavor antitriplet states are detailed in the first part of Table II with the coupling constants $\tilde{c}_{n}$. As can be seen from Table II only the particular term $\tilde{c}_{1}$ keeps the original structure being a product of two quark masses. We identify a single parameter combination $\tilde{c}_{3}$ that probes the product of a quark mass with the second power of some meson mass. The remaining parameters $\tilde{c}_{2}$ and $\tilde{c}_{4}$ select the terms involving the fourth power of a meson mass.
Like in the previously studied cases [1,2] there is a subtle issue as how to treat the flavor singlet structures proportional to $g_{0, \overline{3} \overline{3}]}^{(S)}$ and $b_{1,[\overline{3} \overline{3}]}$. While the first term stems from a chiral symmetric interaction, the second one from a structure that breaks the chiral symmetry explicitly. Nevertheless, the two terms end up with identical tadpole type contributions if the Gell-Mann-Oakes-Renner relations are used. The request of renormalization scale invariance implies that the two contributions in (11) have to be dealt with identically, i.e., we take the replacement

\begin{tabular}{|c|c|c|c|}
\hline$\Sigma_{B}^{(4-\chi)}$ & $B=\Lambda_{c}$ & $B=\Xi_{c}$ & $B=\Xi_{c} \Xi_{c}^{\prime}$ \\
\hline$m_{\pi}^{4}$ & $3 \tilde{c}_{2,[\overline{3} \overline{3}]}+18 \tilde{c}_{4,[\overline{3} \overline{3}]}$ & $3 \tilde{c}_{2,[\overline{3} \overline{3}]}+9 \tilde{c}_{4,[\overline{3} \overline{3}]}$ & $-3 \tilde{c}_{2,[\overline{3} 6]}$ \\
\hline$m_{K}^{4}$ & $4 \tilde{c}_{2,[\overline{3} \overline{3}]}+12 \tilde{c}_{4,[\overline{3} \overline{3}]}$ & $\left.4 \tilde{c}_{2,[\overline{3} \overline{3}]}+18 \tilde{c}_{4,[\overline{3}} \overline{3}\right]$ & $2 \tilde{c}_{2,[\overline{3} 6]}$ \\
\hline$m_{\eta}^{4}$ & $\tilde{c}_{2,[\overline{3} \overline{3}]}+2 \tilde{c}_{4,[\overline{3} \overline{3}]}$ & $\tilde{c}_{2,[\overline{3} \overline{3}]}+5 \tilde{c}_{4,[\overline{3} \overline{3}]}$ & $\tilde{c}_{2,[\overline{3} 6]}$ \\
\hline$B_{0} m m_{\pi}^{2}$ & $18 \tilde{c}_{3,[\overline{3} \overline{3}]}$ & $\left.9 \tilde{c}_{3,[\overline{3}} \overline{3}\right]$ & $-9 \tilde{c}_{1,[\overline{3} 6]}$ \\
\hline$B_{0}\left(m+m_{s}\right) m_{K}^{2}$ & $6 \tilde{c}_{3,[\overline{3} \overline{3}]}$ & $\left.9 \tilde{c}_{3,[\overline{3}} \overline{3}\right]$ & $3 \tilde{c}_{1,[\overline{3} 6]}$ \\
\hline$B_{0} m m_{\eta}^{2}$ & $\left.2 \tilde{c}_{3,[\overline{3}} \overline{3}\right]$ & $\left.\tilde{c}_{3,[\overline{3}} \overline{3}\right]$ & $-\tilde{c}_{1,[\overline{3} 6]}$ \\
\hline$B_{0} m_{s} m_{\eta}^{2}$ & 0 & $4 \tilde{c}_{3,[\overline{3} \overline{3}]}$ & $4 \tilde{c}_{1,[\overline{3} 6]}$ \\
\hline$B_{0}^{2}\left(2 m^{2}+m_{s}^{2}\right)$ & $\tilde{c}_{1,[\overline{3} \overline{3}]}$ & $\tilde{c}_{1,[\overline{3} \overline{3}]}$ & 0 \\
\hline$\Sigma_{B}^{(4-\chi)}$ & $B=\Sigma_{c}$ & $B=\Xi_{c}^{\prime}$ & $B=\Omega_{c}$ \\
\hline $\begin{array}{l}m_{\pi}^{4} \\
m_{K}^{4} \\
m_{\eta}^{4}\end{array}$ & $\begin{array}{c}3 \tilde{c}_{2,[66]}+18 \tilde{c}_{4,[66]}+3 \tilde{c}_{5,[66]} \\
4 \tilde{c}_{2,[66]}+12 \tilde{c}_{4,[66]} \\
\tilde{c}_{2,[66]}+2 \tilde{c}_{4,[66]}+\tilde{c}_{5,[66]}\end{array}$ & $\begin{array}{c}3 \tilde{c}_{2,[66]}+9 \tilde{c}_{4,[66]} \\
4 \tilde{c}_{2,[66]}+18 \tilde{c}_{4,[66]}+6 \tilde{c}_{5,[66]} \\
\tilde{c}_{2,[66]}+5 \tilde{c}_{4,[66]}-2 \tilde{c}_{5,[66]}\end{array}$ & $\begin{array}{c}3 \tilde{c}_{2,[66]} \\
4 \tilde{c}_{2,[66]}+24 \tilde{c}_{4,[66]} \\
\tilde{c}_{2,[66]}+8 \tilde{c}_{4,[66]}+4 \tilde{c}_{5,[66]}\end{array}$ \\
\hline$B_{0} m m_{\pi}^{2}$ & $18 \tilde{c}_{3,[66]}$ & $9 \tilde{c}_{3,[66]}$ & 0 \\
\hline$B_{0}\left(m+m_{s}\right) m_{K}^{2}$ & $6 \tilde{c}_{3,[66]}$ & $9 \tilde{c}_{3,[66]}$ & $12 \tilde{c}_{3,[66]}$ \\
\hline$B_{0} m m_{\eta}^{2}$ & $2 \tilde{c}_{3,[66]}$ & $\tilde{c}_{3,[66]}$ & 0 \\
\hline$B_{0} m_{s} m_{\eta}^{2}$ & 0 & $4 \tilde{c}_{3,[66]}$ & $8 \tilde{c}_{3,[66]}$ \\
\hline$B_{0}^{2}\left(2 m^{2}+m_{s}^{2}\right)$ & $\tilde{c}_{1,[66]}$ & $\tilde{c}_{1,[66]}$ & $\tilde{c}_{1,[66]}$ \\
\hline
\end{tabular}

TABLE II. Contributions to the baryon self-energy proportional to the product of two quark masses are expressed in terms of meson masses as to obtain renormalization scale invariant results. The original form from (8) is recovered in application of the Gell-Mann-Oakes-Renner relations, e.g., $m_{\pi}^{2}=2 B_{0} m$ and $m_{K}^{2}=B_{0}\left(m+m_{s}\right)$. 


$$
g_{0,[\overline{3} \overline{3}]}^{(S)} \rightarrow g_{0,[\overline{3} \overline{3}]}^{(S)}-2 b_{1,[\overline{3} \overline{3}]}
$$

in $G_{B Q}^{(S)}$ but drop the contribution of $b_{1,[\overline{3} \overline{3}]}$ in $G_{B Q}^{(\chi)}$.

Analogous results can be derived for the flavor sextet states with $J^{P}=\frac{1}{2}^{+}$and $J^{P}=\frac{3}{2}^{+}$. Here we detail our derivations for the $J^{P}=\frac{1}{2}^{+}$states without loss of generality. The corresponding expressions for the $J^{P}=\frac{3}{2}^{+}$follow upon the universal substitution $c_{n} \rightarrow e_{n}$. Consider the parameter combinations

$$
\begin{aligned}
\tilde{c}_{1,[66]}= & -\frac{4}{33}\left(33 c_{1,[66]}-45 c_{2,[66]}-15 c_{3,[66]}\right. \\
& \left.+11 c_{4,[66]}-c_{5,[66]}\right), \\
\tilde{c}_{2,[66]}= & -\frac{2}{253}\left(207 c_{2,[66]}+3 c_{3,[66]}-22 c_{4,[66]}+20 c_{5,[66]}\right), \\
\tilde{c}_{3,[66]}= & -\frac{1}{46}\left(9 c_{3,[66]}+26 c_{4,[66]}+14 c_{5,[66]}\right), \\
\tilde{c}_{4,[66]}= & -\frac{1}{92}\left(3 c_{3,[66]}-22 c_{4,[66]}-26 c_{5,[66]}\right), \\
\tilde{c}_{5,[66]}= & -c_{5,[66]},
\end{aligned}
$$

as used in the lower parts of Table II. We point at the oneto-one correspondence of the coefficients in (14) and (16) for all four terms but the $c_{5,[66]}$, which does not have a counterpart in (14). Note that here the replacements

$$
g_{0,[66]}^{(S)} \rightarrow g_{0,[66]}^{(S)}-2 b_{1,[66]} \quad \text { and } \quad b_{1,[66]} \rightarrow 0,
$$

are required in $G_{B Q}^{(S)}$ and $G_{B Q}^{(\chi)}$ respectively.

We turn to the bubble loop contributions properly derived in the subtraction scheme [1,2]. The generic form of the loop contributions can be taken over from our previous works $[1,2,20,21]$. Consider first the contributions to the masses of the $J^{P}=\frac{1}{2}^{+}$states

$$
\begin{aligned}
\bar{\Sigma}_{B \in[\overline{3}, 6]}^{\text {bubble }}= & \sum_{Q \in[8], R \in[\overline{3}, 6]}\left(\frac{G_{Q R}^{(B)}}{2 f}\right)^{2}\left\{-\frac{\left(M_{B}+M_{R}\right)^{2}}{E_{R}+M_{R}} p_{Q R}^{2} \bar{I}_{Q R}\right. \\
& \left.+\frac{M_{R}^{2}-M_{B}^{2}}{2 M_{B}} I_{Q}^{R}+2 \alpha_{Q R}^{(B)}\right\} \\
& +\sum_{Q \in[8], R \in[4]}\left(\frac{G_{Q R}^{(B)}}{2 f}\right)^{2}\left\{-\frac{2}{3} \frac{M_{B}^{2}}{M_{R}^{2}}\left(E_{R}+M_{R}\right) p_{Q R}^{2} \bar{I}_{Q R}\right. \\
& \left.+\frac{\left(M_{R}-M_{B}\right)\left(M_{R}+M_{B}\right)^{3}}{12 M_{B} M_{R}^{2}} I_{Q}^{R}+\frac{4}{3} \alpha_{Q R}^{(B)}\right\},
\end{aligned}
$$

where we encounter the subtraction terms $\alpha_{Q R}^{(B)}$ [see (21)]. We first recall the scalar tadpole and bubble integrals with

$$
\begin{aligned}
I_{Q}^{R}= & \frac{m_{Q}^{2}}{(4 \pi)^{2}} \log \frac{m_{Q}^{2}}{M_{R}^{2}}+\Delta \bar{I}_{Q}^{(0)}, \\
\bar{I}_{Q R}= & \Delta I_{Q R}+\frac{1}{16 \pi^{2}}\left\{\gamma_{B}^{R}-\left(\frac{1}{2}+\frac{m_{Q}^{2}-M_{R}^{2}}{2 M_{B}^{2}}\right) \ln \left(\frac{m_{Q}^{2}}{M_{R}^{2}}\right)\right. \\
& +\frac{p_{Q R}}{M_{B}}\left(\ln \left(1-\frac{M_{B}^{2}-2 p_{Q R} M_{B}}{m_{Q}^{2}+M_{R}^{2}}\right)\right. \\
& \left.\left.-\ln \left(1-\frac{M_{B}^{2}+2 p_{Q R} M_{B}}{m_{Q}^{2}+M_{R}^{2}}\right)\right)\right\}, \\
p_{Q R}^{2}= & \frac{M_{B}^{2}}{4}-\frac{M_{R}^{2}+m_{Q}^{2}}{2}+\frac{\left(M_{R}^{2}-m_{Q}^{2}\right)^{2}}{4 M_{B}^{2}}, \quad E_{R}^{2}=M_{R}^{2}+p_{Q R}^{2},
\end{aligned}
$$

where all finite volume effects are collected into $\Delta \bar{I}_{Q}^{(0)}$ and $\Delta \bar{I}_{Q R}$. For explicit expressions for the latter the reader is

TABLE III. Meson-baryon coupling constants, $G_{Q R}^{(B)}$, in the isospin basis. Only nonvanishing elements are shown for the flavor antitriplet and sextet states with $J^{P}=\frac{1}{2}{ }^{+}$.

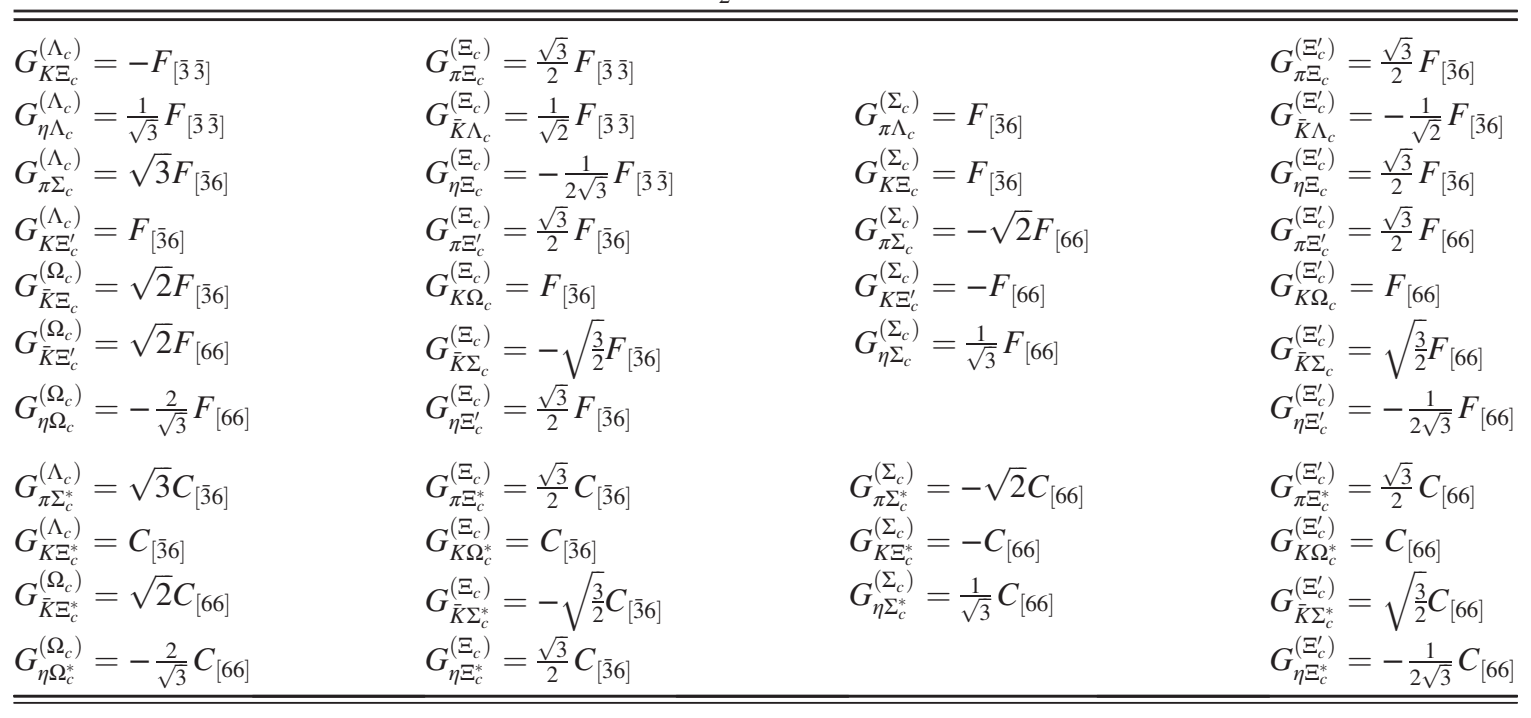


referred to [21]. The sums in (17) extend over the intermediate Goldstone bosons $(Q \in[8])$, the two baryon flavor sextet states with $(R \in[6],[4])$ and one flavor antitriplet $(R \in[\overline{3}])$. The coupling constants $G_{Q R}^{(B)}$ are determined in Tables III and IV by the parameters $F_{[a b]}, D_{[a b]}, C_{[a b]}, H_{[a b]}$ as introduced in (3).
We note that all terms proportional to $m_{Q}^{2 n} \bar{I}_{Q}$ with $n \geq 1$ are dropped in (17) as either higher order or as terms that can be absorbed into our tadpole terms. This requires to use renormalized low-energy parameters $\bar{g}^{(S, V)}$ in (11) of the following form

$$
\begin{aligned}
& \bar{g}_{0,[\overline{3} \overline{3}]}^{(S)}=g_{0,[\overline{3} \overline{3}]}^{(S)}+\frac{1}{3} g_{C,[\overline{3} 6]}^{(S)}, \quad \bar{g}_{D,[\overline{3} \overline{3}]}^{(S)}=g_{D,[\overline{3} \overline{3}]}^{(S)}-g_{C,[\overline{3} 6]}^{(S)}, \\
& \bar{g}_{0,[66]}^{(S)}=g_{0,[66]}^{(S)}, \quad \bar{g}_{1,[66]}^{(S)}=g_{1,[66]}^{(S)}-\frac{2}{3} g_{C,[66]}^{(S)}, \quad \bar{g}_{D,[66]}^{(S)}=g_{D,[66]}^{(S)}-\frac{1}{3} g_{C,[66]}^{(S)}, \\
& \bar{g}_{D,[\overline{3} 6]}^{(S)}=g_{D,[\overline{3} 6]}^{(S)}-\frac{1}{6}\left(\frac{C_{[66]}}{C_{[\overline{3} 6]}} g_{C,[\overline{3} 6]}^{(S)}+\frac{C_{[\overline{3} 6]}}{C_{[66]}} g_{C,[66]}^{(S)}\right), \\
& \bar{g}_{0,[\overline{3} \overline{3}]}^{(V)}=g_{0,[\overline{3} \overline{3}]}^{(V)}+\frac{1}{3} g_{C,[\overline{3} 6]}^{(V)}, \quad \bar{g}_{1,[\overline{3} \overline{3}]}^{(V)}=g_{1,[\overline{3} \overline{3}]}^{(V)}, \quad \bar{g}_{D,[\overline{3} \overline{3}]}^{(V)}=g_{D,[\overline{3} \overline{3}]}^{(V)}-g_{C,[\overline{3} 6]}^{(V)}, \\
& \bar{g}_{0,[66]}^{(V)}=g_{0,[66]}^{(V)}, \quad \bar{g}_{1,[66]}^{(V)}=g_{1,[66]}^{(V)}-\frac{2}{3} g_{C,[66]}^{(V)}, \quad \bar{g}_{D,[66]}^{(V)}=g_{D,[66]}^{(V)}-\frac{1}{3} g_{C,[66]}^{(V)}, \\
& \bar{g}_{D,[\overline{3} 6]}^{(V)}=g_{D,[\overline{3} 6]}^{(V)}-\frac{1}{6}\left(\frac{C_{[66]}}{C_{[\overline{3} 6]}} g_{C, \overline{3} 6]}^{(V)}+\frac{C_{[\overline{3} 6]}}{C_{[66]}} g_{C,[66]}^{(V)}\right), \\
& g_{C,[a 6]}^{(S)}=\frac{C_{[a 6]}^{2}}{2 M_{[a]}} \frac{4 M_{[a]}^{2}+\Delta_{[a]} M_{[a]}-\Delta_{[a]}^{2}}{4\left(M_{[a]}+\Delta_{[a]}\right)^{2}}, \quad g_{C,[a 6]}^{(V)}=\frac{C_{[a 6]}^{2}}{4 M_{[a]}^{2}}\left(\frac{M_{[a]}}{M_{[a]}+\Delta_{[a]}}\right)^{2},
\end{aligned}
$$

with the chiral limit mass spin splitting values $\Delta_{[a]}=$ $M_{[4]}-M_{[a]}$ for $a=\overline{3}, 6$. We use here the notation for the flavor SU(3) chiral limit baryon masses $M_{B}^{(0)} \leftrightarrow M_{[a]}$ as introduced with (10).

Given our approach the scalar bubble loop function $\bar{I}_{Q R}$ does not depend on the renormalization scale $\mu$. We point the reader at the subtraction terms $\gamma_{B}^{R}$ and $\alpha_{Q R}^{(B)}$ in (17). It is recalled that the subtraction

$$
\gamma_{B}^{R}=-\lim _{m, m_{s} \rightarrow 0} \frac{M_{R}^{2}-M_{B}^{2}}{M_{B}^{2}} \log \left|\frac{M_{R}^{2}-M_{B}^{2}}{M_{R}^{2}}\right|,
$$

makes sure that the scalar bubble $\bar{I}_{Q R}\left(M_{B}\right)$ will vanish in the chiral limit with $m_{Q} \rightarrow 0$ strictly. This protects the tree level slope parameters $b_{n,[a b]}$ in (5) as advocated above. The additional term $\alpha_{Q R}^{(B)}$ is required to protect a chiral theorem. There are nonanalytic terms proportional to $m_{Q}^{3}$ that arise from the bubble loop contributions. Only in the presence of the subtraction terms $\alpha_{Q R}^{(B)}$ they take their proper form. From $[1,2]$ it is recalled

$$
\begin{aligned}
\alpha_{Q R}^{(B)} & =\frac{\alpha_{1} \Delta^{2}}{(4 \pi)^{2}}\left(M_{R}-M_{B}-\Delta_{B}\right)\left(\frac{\Delta \partial}{\partial \Delta}+1\right) \gamma_{1}+\frac{\Delta m_{Q}^{2}}{(4 \pi)^{2}} \alpha_{1} \gamma_{2}, \\
M & =M_{B}^{(0)}, \quad \Delta=M_{R}^{(0)}-M_{B}^{(0)}, \quad \gamma_{1}=\frac{2 M+\Delta}{2 M} \log \frac{\Delta^{2}(2 M+\Delta)^{2}}{(M+\Delta)^{4}}, \\
\Delta_{B} & =\Delta \frac{M_{B}}{M}, \quad \gamma_{2}=-\frac{2 M^{2}+2 \Delta M+\Delta^{2}}{2 M(2 M+\Delta)} \log \frac{\Delta^{2}(2 M+\Delta)^{2}}{(M+\Delta)^{4}}-\frac{M}{2 M+\Delta}, \\
\text { with } \quad \alpha_{1} & =\frac{(2 M+\Delta)^{4}}{16 M^{2}(M+\Delta)^{2}} \quad \text { if } R \in[4] \quad \text { but } \alpha_{1}=\frac{(2 M+\Delta)^{2}}{4 M^{2}} \quad \text { if } R \in[\overline{3}, 6],
\end{aligned}
$$


TABLE IV. Meson-baryon coupling constants, $G_{Q R}^{(B)}$, in the isospin basis. Only nonvanishing elements are shown for $B \in[4]$, i.e., the flavor sextet states with $J^{P}=\frac{3}{2}^{+}$.

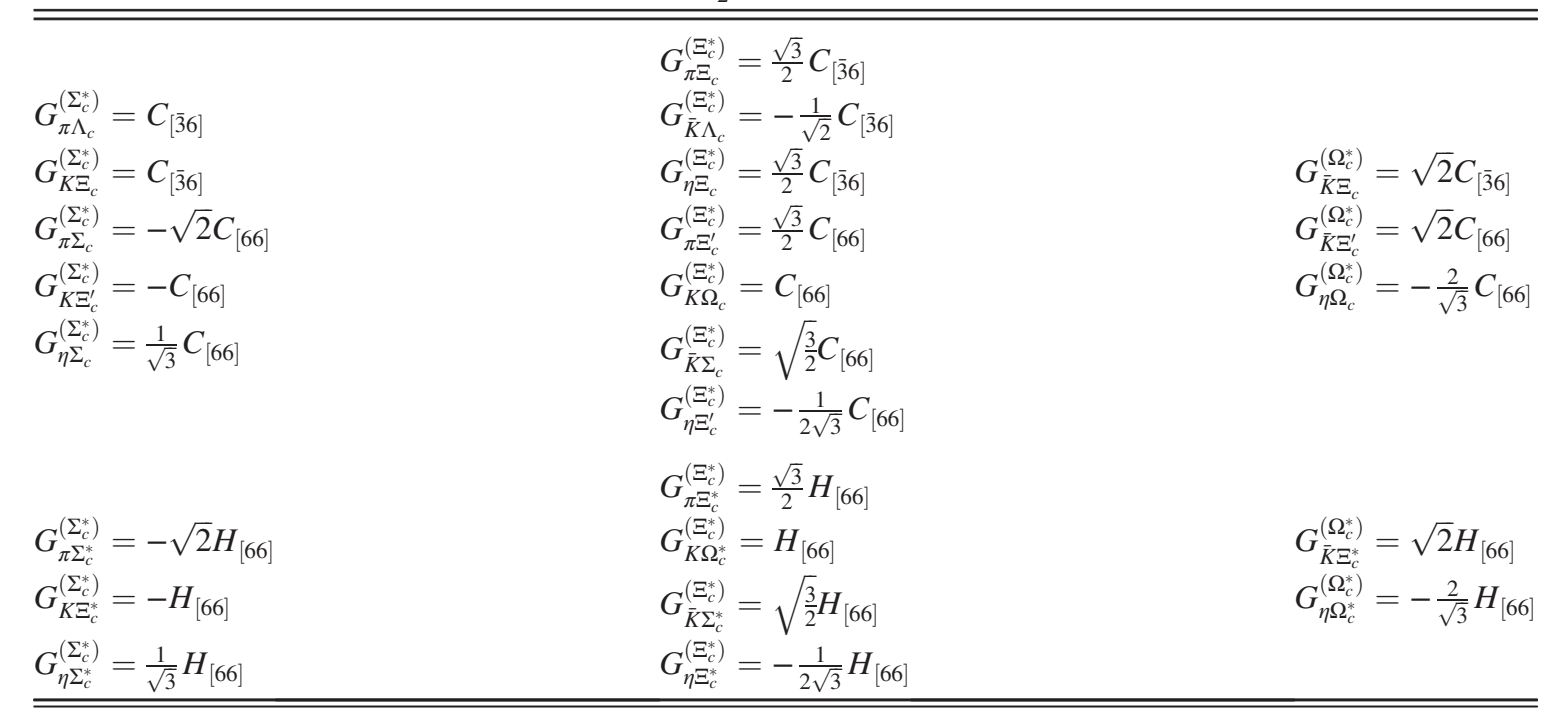

where we note that the last expression for $\alpha_{1}$ in (21) was not needed in [1] since there only one flavor multiplet of baryon states with $J^{P}=\frac{1}{2}+$ occurs.

We close this section with the bubble loop contribution for the $J^{P}=\frac{3}{2}^{+}$states. Again the form for the loop contributions can be inferred from our previous work $[1,20]$. We find

$$
\begin{aligned}
\bar{\Sigma}_{B \in[4]}^{\text {bubble }}= & \sum_{Q \in[8], R \in[\overline{3}, 6]}\left(\frac{G_{Q R}^{(B)}}{2 f}\right)^{2}\left\{-\frac{1}{3}\left(E_{R}+M_{R}\right) p_{Q R}^{2} \bar{I}_{Q R}\right. \\
& \left.+\frac{\left(M_{R}-M_{B}\right)\left(M_{R}+M_{B}\right)^{3}}{24 M_{B}^{3}} I_{Q}^{R}+\frac{2}{3} \alpha_{Q R}^{(B)}\right\} \\
& +\sum_{Q \in[8], R \in[4]}\left(\frac{G_{Q R}^{(B)}}{2 f}\right)^{2}\left\{-\frac{\left(M_{B}+M_{R}\right)^{2}}{9 M_{R}^{2}} \frac{2 E_{R}\left(E_{R}-M_{R}\right)+5 M_{R}^{2}}{E_{R}+M_{R}} p_{Q R}^{2} \bar{I}_{Q R}\right. \\
& \left.+\frac{M_{R}^{4}+M_{B}^{4}+12 M_{R}^{2} M_{B}^{2}-2 M_{R} M_{B}\left(M_{B}^{2}+M_{R}^{2}\right)}{36 M_{B}^{3} M_{R}^{2}}\left(M_{R}^{2}-M_{B}^{2}\right) I_{Q}^{R}\right\}
\end{aligned}
$$

with the Clebsch $G_{Q R}^{(B)}$ listed in Table IV. The renormalization of the coupling constants $\bar{h}^{(S, V)}$ from the bubble-loop diagram is

$$
\begin{aligned}
& \bar{h}_{n,[66]}^{(S)}=h_{n,[66]}^{(S)} \quad \text { for } n=0,1,3,5, \\
& \bar{h}_{2,[66]}^{(S)}=h_{2,[66]}^{(S)}+\frac{1}{6} h_{C,[\overline{3} 6]}^{(S)}+\frac{1}{6} h_{C,[66]}^{(S)} \quad \bar{h}_{4,[66]}^{(S)}=h_{4,[66]}^{(S)}-\frac{1}{3} h_{C,[\overline{3} 6]}^{(S)}+\frac{1}{3} h_{C,[66]}^{(S)}, \\
& \bar{h}_{0,[66]}^{(V)}=h_{0,[66]}^{(V)}, \quad \bar{h}_{1,[66]}^{(V)}=h_{1,[66]}^{(V)}-\frac{H_{[66]}^{2}}{9 M_{[4]}^{2}}+\frac{1}{3}\left(h_{C,[\overline{3} 6]}^{(V)}-h_{C,[66]}^{(V)}\right), \\
& \bar{h}_{2,[66]}^{(V)}=h_{2,[66]}^{(V)}-\frac{H_{[66]}^{2}}{18 M_{[4]}^{2}}-\frac{1}{6}\left(h_{C,[\overline{3} 6]}^{(V)}+h_{C,[66]}^{(V)}\right), \\
& h_{C,[a 6]}^{(S)}=\frac{C_{[a 6]}^{2}}{2 M_{[a]}} \frac{4 M_{[a]}^{3}+5 \Delta_{[a]} M_{[a]}^{2}+2 \Delta_{[a]}^{2} M_{[a]}}{4\left(M_{[a]}+\Delta_{[a]}\right)^{3}}, \quad h_{C,[a 6]}^{(V)}=\frac{C_{[a 6]}^{2}}{4 M_{[4]} M_{[a]}} \frac{M_{[a]}}{M_{[a]}+\Delta_{[a]}},
\end{aligned}
$$


with again $\Delta_{[a]}=M_{[4]}-M_{[a]}$ for $a=\overline{3}, 6$. It is left to detail the subtraction term $\alpha_{Q R}^{(B)}$ for the $J^{P}=\frac{3}{2}^{+}$states which takes the form

$$
\begin{aligned}
\alpha_{Q R}^{(B)} & =\frac{\beta_{1} \Delta^{2}}{(4 \pi)^{2}}\left(M_{B}-M_{R}-\Delta_{B}\right)\left(\frac{M+\Delta}{M}\right)\left(\frac{\Delta \partial}{\partial \Delta}+1\right) \delta_{1}+\frac{\Delta m_{Q}^{2}}{(4 \pi)^{2}} \beta_{1} \delta_{2}, \\
M & =M_{R}^{(0)}, \quad \Delta=M_{B}^{(0)}-M_{R}^{(0)} \quad \delta_{1}=-\frac{M(2 M+\Delta)}{(M+\Delta)^{2}} \log \left|\frac{\Delta(2 M+\Delta)}{M^{2}}\right|, \\
\Delta_{B} & =\Delta \frac{M_{B}}{M+\Delta}, \quad \delta_{2}=\frac{M}{2 M+\Delta}+M \frac{2 M^{2}+2 \Delta M+\Delta^{2}}{(2 M+\Delta)(M+\Delta)^{2}} \log \left|\frac{\Delta(2 M+\Delta)}{M^{2}}\right|,
\end{aligned}
$$

with $\quad \beta_{1}=\frac{(2 M+\Delta)^{4}}{16 M(M+\Delta)^{3}}$.

For a more in depth discussion of the various arguments in favor of the applied renormalization scheme we refer to our previous works $[1,2]$.

\section{LARGE- $N_{c}$ SUM RULES AT THE ONE-LOOP LEVEL}

In our previous work [11] we derived sum rules for our low-energy constants as they arise in QCD with a large number of colors $\left(N_{c}\right)$. The analysis was performed for tree-level expressions derived from the chiral Lagrangian. As was pointed out already for the analogous case of a study for the baryon octet and decuplet masses [1], such relations need to be supplemented by constraints that are implied by the renormalization scale invariance condition.

This is readily understood if one considers the scale dependence of the symmetry breaking counter terms proportional to $c_{i}$ and $e_{i}$ of (8). The request that their contributions to the baryon masses are renormalization scale invariant is readily derived with

$$
\begin{aligned}
\mu^{2} \frac{\mathrm{d}}{\mathrm{d} \mu^{2}} c_{i,[a b]} & =-\frac{1}{4} \frac{1}{(4 \pi f)^{2}} \Gamma_{c_{i,[a b]}}, \\
\mu^{2} \frac{\mathrm{d}}{\mathrm{d} \mu^{2}} e_{i,[66]} & =-\frac{1}{4} \frac{1}{(4 \pi f)^{2}} \Gamma_{e_{i,[6]]}},
\end{aligned}
$$

where all $\Gamma_{c_{i,[a b]}}$ and $\Gamma_{e_{i, a b]}}$ are detailed in Appendix B. They depend on the symmetry conserving two-body terms $g$ and $h$ in (7), but also on the symmetry breaking parameters $b$ and $d$ in (4). In turn if we insist on the leading order sum rules for the $c_{i}$ and $e_{i}$ of Appendix A the following conditions arise

$$
\begin{array}{ll}
\Gamma_{c_{n,[66]}}=\Gamma_{e_{n,[66]}} & \Gamma_{c_{n,[\overline{3}]}}=0 \quad \text { for all } n, \\
\Gamma_{c_{1,[\overline{3} \overline{3}]}}=\Gamma_{c_{1,[66]}}, & \Gamma_{c_{2,[\overline{3}]}}=\Gamma_{c_{2,[66]}}, \\
\Gamma_{c_{3,[\overline{3} \overline{3}]}}=\Gamma_{c_{3,[66]}}, & \Gamma_{c_{4,[\overline{3}]}}=\Gamma_{c_{4,[66]}}-\Gamma_{c_{5,[66]}} .
\end{array}
$$

If supplemented by the leading order sum rules for the remaining low-energy constants we arrive at the additional relations

$$
\begin{aligned}
\bar{g}_{1,[66]}^{(S)} & =2 \bar{g}_{0,[\overline{3} \overline{3}]}^{(S)}=-\frac{1}{4} M_{1,[66]}^{(V)} \quad \text { with } \\
M & =M_{[\overline{4}]}=M_{[6]}=M_{[\overline{3}]} \quad \text { or } \quad \bar{g}_{1,[66]}^{(V)}=0=\bar{g}_{D,[66]}^{(V)} .
\end{aligned}
$$

This is an amazing prediction since now altogether we have $40=36+4$ sum rules at leading order. Thus from the 54 low-energy constants we started out, there remain only $5=$ $14-8-1$ parameters that we have to adjust to the QCD lattice data set on the charmed baryon masses. In our parameter count we subtract the 8 charmed baryon masses known from the PDG and one axial coupling constant $C_{[\overline{3} 6]}$ which is determined by the empirically known decay process $\Sigma_{c}^{++}(2520) \rightarrow \Lambda_{c}^{+} \pi^{+}$.

We close this section by a study of such sum rules at subleading order in the $1 / N_{c}$ expansion. From Appendix A we obtain the following conditions

$$
\begin{aligned}
& \Gamma_{c_{1,[66]}}=\Gamma_{e_{1,[6]}}, \quad \Gamma_{c_{2,[6]}}=\Gamma_{e_{2,[66]}} \quad \Gamma_{c_{1,[\overline{3} 3]}}+\Gamma_{c_{2,[\overline{3} 3]}}=\Gamma_{c_{1,[66]}}+\Gamma_{c_{2,[66]}}, \\
& \Gamma_{c_{1,[\overline{3} 6]}}=5 \sqrt{3}\left(\Gamma_{c_{3,[66]}}-\Gamma_{e_{3,[6]}}\right), \quad \Gamma_{c_{2,[\overline{3} 6]}}=5 \sqrt{3}\left(\Gamma_{c_{4,[6]}}-\Gamma_{e_{4,[6]}}\right),
\end{aligned}
$$


which we supplement by sum rules for low-energy constants valid at subleading order. This leads to the following five additional conditions

$$
\begin{aligned}
\bar{g}_{0,[66]}^{(V)}= & \frac{b_{2,[66]}-d_{2,[66]}}{M_{[6]}-M_{[4]}} \frac{48 M_{[6]}}{163\left(M_{[6]}+M_{[4]}\right)}, \\
\bar{g}_{D,[66]}^{(S)}= & \bar{h}_{2,[66]}^{(S)}+\frac{\left(M_{[\overline{3}]}+M_{[6]}\right)^{2}\left(M_{[4]}-M_{[6]}\right)\left(M_{[4]}+M_{[6]}\right)}{4 M_{[4]}\left(M_{[\overline{3}]}-M_{[6]}\right)\left(M_{[\overline{3}]}+3 M_{[6]}\right)} \bar{h}_{2,[66]}^{(V)}+\frac{b_{2,[66]}-d_{2,[66]}}{M_{[\overline{[}]}-M_{[6]}} \frac{99\left(M_{[\overline{3}]}^{2}+2 M_{[\overline{3}]} M_{[6]}-59 M_{[6]}^{2}\right)}{163\left(M_{[\overline{3}]}+3 M_{[6]}\right)} \\
\bar{g}_{D,[66]}^{(V)}= & \frac{M_{[6]}\left(M_{[\overline{3}]}-2 M_{[4]}+M_{[6]}\right)\left(M_{[\overline{3}]}+2 M_{[4]}+M_{[6]}\right)}{M_{[4]}\left(M_{[\overline{3}]}-M_{[6]}\right)\left(M_{[\overline{3}]}+3 M_{[6]}\right)} \bar{h}_{2,[66]}+\frac{b_{2,[66]}-d_{2,[6]]}}{M_{[\overline{3}]}-M_{[6]}} \frac{22176 M_{[6]}}{163\left(M_{[\overline{3}]}+3 M_{[6]}\right)} \\
M_{[4]} \bar{h}_{1,[66]}^{(V)}= & 78 \bar{g}_{0,[\overline{3} \overline{3}]}^{(S)}+35 \bar{g}_{D,[\overline{3} \overline{3}]}^{(S)}-78 \bar{g}_{0,[66]}^{(S)}-35 \bar{h}_{2,[66]}^{(S)}-4 \bar{h}_{4,[66]}^{(S)}-\bar{h}_{5,[66]}^{(S)}+\frac{M_{[\overline{3}]}}{4}\left(78 \bar{g}_{0,[\overline{3} \overline{3}]}^{(V)}+8 \bar{g}_{1,[\overline{3} \overline{3}]}^{(V)}+35 \bar{g}_{D,[\overline{3} \overline{3}]}^{(V)}\right)-\frac{35 M_{[4]}}{4} \bar{h}_{2,[66]}^{(V)} \\
& -27\left(b_{2,[\overline{3} \overline{3}]}-d_{2,[66]}\right)-\frac{b_{2,[66]}-d_{2,[66]}}{M_{[6]}-M_{[4]}} \frac{936 M_{[4]}^{2}}{163\left(M_{[6]}+M_{[4]}\right)} \\
M_{[6]} \bar{g}_{1,[66]}^{(V)}= & M_{[4]} \bar{h}_{1,[66]}^{(V)}-4 \bar{g}_{1,[66]}^{(S)}+4 \bar{h}_{4,[66]}^{(S)}+\bar{h}_{5,[66]}^{(S)} .
\end{aligned}
$$

Superficially the expressions (29) appear singular at either $M_{[6]} \rightarrow M_{[4]}$ or $M_{[6]} \rightarrow M_{[\overline{3}]}$. However this is not the case since in the later limits there are additional relations that ensure that all low-energy constants remain finite in those limits. We remind the reader of the sum rules that arise in large- $N_{c}$ QCD at leading order with $M_{[6]}=M_{[4]}=M_{[\overline{3}]}$. Since the heavy-quark mass limit leads to $M_{[6]}=M_{[4]}$ only, it follows the scaling relation

$$
b_{2,[66]}-d_{2,[66]} \sim\left(M_{[6]}-M_{[4]}\right)\left(M_{[6]}-M_{[\overline{3}]}\right),
$$

which implies that the low-energy parameters in (29) remain finite in the latter two limits. More specifically, the first three identities in (29) approach the leading order large- $N_{c}$ relations $\bar{g}_{0,[66]}^{(V)}=0$ and $\bar{g}_{D,[66]}^{(S)}=\bar{h}_{2,[66]}^{(S)}$ and $\bar{g}_{D,[66]}^{(V)}=\bar{h}_{2,[66]}^{(V)}$ of Appendix A. The remaining two identities recover the two scale relations $\bar{g}_{1,[66]}^{(S)}=2 \bar{g}_{0,[\overline{3} \overline{3}]}^{(S)}=$ $-\frac{1}{4} M \bar{g}_{1,[66]}^{(V)}$ in this limit with $M=M_{[6]}=M_{[4]}=M_{[\overline{3}]}$ [see Eq. (27)]. Note a subtle issue concerning the order at which the two limits $M_{c} \rightarrow \infty$ and $N_{c} \rightarrow \infty$ have to be applied. Consistent results follow only if the heavy-quark mass limit with $M_{[6]} \rightarrow M_{[4]}$ at $M_{[\overline{3}]} \neq M_{[4]}$ is applied first.

At subleading order altogether we have $22=17+5$ sum rules. Thus from the 54 low-energy constants, there remain only $23=32-8-1$ parameters that we have to adjust to the QCD lattice data set on the charmed baryon masses. Even at subleading order we deem this to be a significant result which paves the way toward a quantitative and controlled approach to chiral dynamics of charmed baryons.

\section{A CONVERGENCE STUDY FOR THE BUBBLE LOOP}

The purpose of the following section is to decompose the

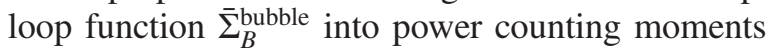

$$
\bar{\Sigma}_{B}^{\text {bubble }}=\bar{\Sigma}_{B}^{\text {bubble }-3}+\bar{\Sigma}_{B}^{\text {bubble-4 }}+\bar{\Sigma}_{B}^{\text {bubble }-5}+\cdots,
$$

and illustrate the convergence properties of such an expansion at hand of the physical meson and baryon masses. It is emphasized that any conventional chiral expansion in terms of bare meson and baryon masses appears futile at physical up, down and strange quark masses, at least for the baryon masses with zero charm content. From our previous study of the chiral expansion for the charm meson masses [2] we already learned that such a conventional strategy appears ill defined even for charmed systems. Though, a conventional expansion for the charm baryon masses may not be as disastrous as it is for the baryons with zero charm content, we anticipate that our expansion in terms of on-shell masses generates much more useful and convincing results.

Given our framework and notations the required expressions can be readily deduced from our previous work [1], where however a slight adaptation is necessary. Any of the moments in (31) receives three types of contributions

$$
\bar{\Sigma}_{B \in[a]}^{\text {bubble-n }}=\bar{\Sigma}_{B \in[a],[\overline{3}]}^{\text {bubble-n }}+\bar{\Sigma}_{B \in[a],[6]}^{\text {bubble-n }}+\bar{\Sigma}_{B \in[a],[4]}^{\text {bubble-n }},
$$

which are classified according to the flavor or spin multiplicity of the intermediate charmed baryon states. We will exemplify such results for the leading order term in the expansion. 
For the spin-three-half baryons in the flavor sextet we write

$$
\begin{aligned}
\Sigma_{B \in[4],[4]}^{\text {bubble }-3}= & \sum_{Q \in[8], R \in[4]}\left(\frac{1}{4 \pi f} G_{Q R}^{(B)}\right)^{2} \frac{5}{9}\left\{\frac{m_{Q}^{2}}{2 M_{B}}\left(1-\log \frac{m_{Q}}{M_{R}}\right)-\frac{\pi}{2} m_{Q}\right\}\left(m_{Q}^{2}-\left(M_{R}-M_{B}\right)^{2}\right), \\
\bar{\Sigma}_{B \in[4],[\overline{3}]}^{\text {bubble } 3}= & \sum_{Q \in[8], R \in[\overline{3}]}\left(\frac{1}{4 \pi f} G_{Q R}^{(B)}\right)^{2} \frac{\beta_{1}}{6}\left\{\hat{\delta}_{2} \Delta m_{Q}^{2}+\left[\delta_{1} \Delta_{B}-\tilde{\delta}_{1}\left(M_{B}-M_{R}\right)\right] \Delta_{Q}^{2}-\hat{\delta}_{1} \Delta^{2}\left(M_{R}-M_{B}+\Delta_{B}\right)\right. \\
& +\frac{(2 M+\Delta) M}{2(M+\Delta)^{2}}\left[\left(\Delta_{Q}^{2}-\frac{1}{2} m_{Q}^{2}\right)\left(M_{B}-M_{R}\right) \log \frac{m_{Q}^{2}}{M_{R}^{2}}+\Delta_{Q}^{3}\left(\log \left(M_{R}-M_{B}-\Delta_{Q}\right)-\log \left(M_{R}-M_{B}+\Delta_{Q}\right)\right)\right] \\
& \left.+\frac{m_{Q}^{2}}{\Delta_{B}}\left(-\tilde{\delta}_{2} \Delta_{Q}^{2}+\tilde{\delta}_{3} m_{Q}^{2} \log \frac{m_{Q}^{2}}{M_{R}^{2}}\right)\right\}, \\
\Delta_{Q}= & {\left[\left(M_{B}-M_{R}\right)^{2}-m_{Q}^{2}\right]^{1 / 2}, \quad \Delta_{B}=\Delta \frac{M_{B}}{M+\Delta}, } \\
\hat{\delta}_{1}= & \frac{(2 M+\Delta)}{2 M} \frac{\partial}{\partial \Delta} \frac{2(M+\Delta)}{2 M+\Delta}\left(\delta_{1}-\tilde{\delta}_{1}\right)+\tilde{\delta}_{1}, \\
\hat{\delta}_{2}= & \delta_{2}+\frac{1}{2}\left(\delta_{1}-\tilde{\delta}_{1}\right) \frac{\Delta^{2}}{(2 M+\Delta)^{2}}, \quad \tilde{\delta}_{1}=\delta_{1}+\frac{M(2 M+\Delta)}{(M+\Delta)^{2}} \ln \frac{2|\Delta|}{M},
\end{aligned}
$$

where $\Delta=M_{[4]}-M_{[\overline{3}]}$ and $M=M_{[\overline{3}]}$ in this case. Note that the coefficients $\beta_{1}$ and $\delta_{1}, \delta_{2}$ we encountered already in the definitions of the subtraction terms $\alpha_{Q R}^{(B)}$ in (24). A complete collection of such coefficients is provided in Appendix B of [1].

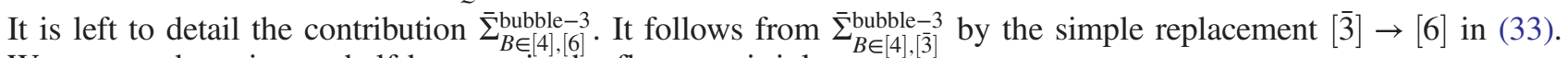

We turn to the spin-one-half baryons in the flavor antitriplet

$$
\begin{aligned}
\bar{\Sigma}_{B \in[\overline{3}],[\overline{3}]}^{\text {bubble } 3}= & \sum_{Q \in[8], R \in[\overline{3}]}\left(\frac{1}{4 \pi f} G_{Q R}^{(B)}\right)^{2}\left\{\frac{m_{Q}^{2}}{2 M_{B}}\left(1-\log \frac{m_{Q}}{M_{R}}\right)-\frac{\pi}{2} m_{Q}\right\}\left(m_{Q}^{2}-\left(M_{R}-M_{B}\right)^{2}\right), \\
\bar{\Sigma}_{B \in[\overline{3}],[4]}^{\text {bubble }-3}= & \sum_{Q \in[8], R \in[4]}\left(\frac{1}{4 \pi f} G_{Q R}^{(B)}\right)^{2} \frac{\alpha_{1}}{3}\left\{\hat{\gamma}_{2} \Delta m_{Q}^{2}+\left[\gamma_{1} \Delta_{B}-\tilde{\gamma}_{1}\left(M_{R}-M_{B}\right)\right] \Delta_{Q}^{2}+\hat{\gamma}_{1} \Delta^{2}\left(M_{R}-M_{B}-\Delta_{B}\right)\right. \\
& -\frac{2 M+\Delta}{2 M}\left[\left(\Delta_{Q}^{2}-\frac{1}{2} m_{Q}^{2}\right)\left(M_{R}-M_{B}\right) \log \frac{m_{Q}^{2}}{M_{R}^{2}}+\Delta_{Q}^{3}\left(\log \left(M_{R}-M_{B}+\Delta_{Q}\right)-\log \left(M_{R}-M_{B}-\Delta_{Q}\right)\right)\right] \\
& +\frac{m_{Q}^{2}}{\Delta_{B}}\left[-\tilde{\gamma}_{2} \Delta_{Q}^{2}+\tilde{\gamma}_{3} m_{Q}^{2} \log \frac{m_{Q}^{2}}{\left.\left.M_{R}^{2}\right]\right\},}\right. \\
\Delta_{Q}= & {\left[\left(M_{B}-M_{R}\right)^{2}-m_{Q}^{2}\right]^{1 / 2}, \quad \Delta_{B}=\Delta \frac{M_{B}}{M}, } \\
\hat{\gamma}_{1}= & \frac{2 M+\Delta}{2 M} \frac{\partial}{\partial \Delta} \frac{2 \Delta M}{2 M+\Delta}\left(\gamma_{1}-\tilde{\gamma}_{1}\right)+\tilde{\gamma}_{1}, \\
\hat{\gamma}_{2}= & \gamma_{2}+\frac{1}{2}\left(\gamma_{1}-\tilde{\gamma}_{1}\right) \frac{\Delta^{2}}{(2 M+\Delta)^{2}}, \quad \tilde{\gamma}_{1}=\gamma_{1}-\frac{2 M+\Delta}{M} \ln \frac{2|\Delta|}{M+\Delta},
\end{aligned}
$$

where $\Delta=M_{[4]}-M_{[\overline{3}]}$ and $M=M_{[\overline{3}]}$. The dimension less coefficients $\alpha_{1}$ and $\gamma_{1}, \gamma_{2}$ are detailed not only in (21) but also in Appendix A of [1]. They depend on the ratio $\Delta / M$ only. In this case the missing term $\bar{\Sigma}_{B \in[\overline{3}],[6]}^{\text {bubble-3}}$ can be obtained from $\bar{\Sigma}$ bubble-3 with

$$
\bar{\Sigma}_{B \in[\overline{3}],[6]}^{\text {bubble-3 }}=\frac{3}{2} \bar{\Sigma}_{B \in[\overline{3}],[4]}^{\text {bubble-3 }} \quad \text { with } \quad \Delta=M_{[6]}-M_{[\overline{3}]} \quad \text { and } \quad M=M_{[\overline{3}]},
$$

where, however, one must use $\alpha_{1}=(2 M+\Delta)^{2} /\left(4 M^{2}\right)$ as given already in (21).

It remains to detail the chiral decomposition for the masses of the spin-one-half baryons in the flavor sextet. The terms $\bar{\Sigma}$ bubble-3 and $\bar{\Sigma}_{B \in[6],[6]}^{\text {bubble-3 } 3}$ follow directly from (34) by the overall replacement $[\overline{3}] \rightarrow[6]$. Then, the missing term 
$\bar{\Sigma}_{B \in[6],[3]}^{\text {buble-3 }}$ is obtained from $(3 / 2) \bar{\Sigma}_{B \in[6],[4]}^{\text {buble-3 }}$ by the identifications $\Delta=-M_{[6]}+M_{[\overline{3}]}<0, M=M_{[6]}$ together with $\alpha_{1}=$ $(2 M+\Delta)^{2} /\left(4 M^{2}\right)$.

With the construction of the third order terms (33), (34) it is straightforward to correctly identify the corresponding fourth and fifth order terms from [1]. Note that the higher order terms involve additional coefficients $\alpha_{n}, \tilde{\alpha}_{n}$ and $\gamma_{n}, \tilde{\gamma}_{n}$ and $\beta_{n}, \tilde{\beta}_{n}$ and $\delta_{n}, \tilde{\delta}_{n}$ that are detailed at the beginnings of Appendices A and B of our previous work [1]. All such coefficients are dimension less and depend on the ratio $\Delta / M$ only. It should be noted that if the ratio $\Delta / M$ turns out to be significantly smaller than $1 / 3$ a further expansion of our results in powers of such a ratio may be justified. However, this can be decided only after a full analysis of the lattice data set has been performed.

\section{SOME NUMERICAL RESULTS}

We now generate some numerical results illustrating the convergence properties of the chiral expansion. Since the relevant set of low-energy parameters is basically unknown we focus on the chiral decomposition of the one-loop bubble functions as detailed in the previous chapter.

Any numerical estimate requires the values of the onshell baryon and meson masses involved. Those we take from the PDG [22]. While for any of the hadron masses $m_{Q}$ or $M_{B}, M_{R}$ we apply an isospin average to the values of the PDG [22], for the chiral limit masses $M_{[\overline{3}]}, M_{[6]}$ and $M_{[4]}$ we take the flavor $\mathrm{SU}(3)$ average of the corresponding multiplet masses from the PDG. The latter assumption is ad-hoc and constitutes a zeroth order estimate for such values only.

It is left to set the axial-vector coupling constants $F$ and $C$. In [10] the estimates $F_{[\overline{3} 6]} \simeq 0.82$ and $C_{[\overline{3} 6]} \simeq 1.36$ were derived from the hadronic decay widths of spin-one-half $\Sigma_{c}^{++}(2455)$ and spin-three-half $\Sigma_{c}^{++}(2520)$ baryons. We provide an update of such values as is implied by the latest decay widths claimed in the PDG [22]. We confirm that

$$
\begin{gathered}
\Gamma_{\Sigma_{c}^{++}(2455) \rightarrow \Lambda_{c}^{+} \pi^{+}}=1.89_{-0.18}^{+0.09} \mathrm{MeV} \rightarrow F_{[\overline{3} 6]}=0.753_{-0.037}^{+0.018}, \\
\Gamma_{\Sigma_{c}^{++}(2520) \rightarrow \Lambda_{c}^{+} \pi^{+}}=14.78_{-0.40}^{+0.30} \mathrm{MeV} \rightarrow C_{[\overline{3} 6]}=1.378_{-0.019}^{+0.014}, \\
\Gamma_{\Sigma_{c}^{0}(2520) \rightarrow \Lambda_{c}^{+} \pi^{-}}=15.30_{-0.50}^{+0.40} \mathrm{MeV} \rightarrow C_{[\overline{3} 6]}=1.401_{-0.023}^{+0.018},
\end{gathered}
$$

translate into estimates for $F_{[\overline{3} 6]}$ and $C_{[\overline{3} 6]}$ which are compatible with the large- $N_{c}$ relation $C_{[\overline{3} 6]}=\sqrt{3} F_{[\overline{3} 6]}$ at the $5 \%$ level. In the following we use the leading order relations (A1). This leaves undetermined the axial-coupling constant $F_{[66]}$ only.

We consider two scenarios, in the first one we use the value $C_{[\overline{3} 6]}=1.35$ together with $F_{[66]}=0$, in the second one $C_{[\overline{3} 6]}=0$ with $F_{[66]}=1$. Once a value for $F_{[66]}$ is known the physical self-energies can be reconstructed unambiguously in terms of our decomposition into the
TABLE V. Baryon self-energies evaluated with physical meson and baryon masses using the leading order large- $N_{c}$ relations for the axial vector coupling constants (A1). The table collects all contributions of our first scenario with $C_{[\overline{3} 6]}=1.35=\sqrt{3} F_{[\overline{3} 6]}$ and $F_{[66]}=F_{[\overline{3} \overline{3}]}=C_{[66]}=H_{[66]}=0$.

\begin{tabular}{lrrrrr}
\hline \hline B & $\bar{\Sigma}_{B}^{\text {bubble }}$ & $\bar{\Sigma}_{B}^{\text {bubble-(3+4+5) }}$ & $\bar{\Sigma}_{B}^{\text {bubble-3 }}$ & $\bar{\Sigma}_{B}^{\text {bubble-4 }}$ & $\bar{\Sigma}_{B}^{\text {bubble-5 }}$ \\
\hline$\Lambda_{c}$ & -146.20 & -146.24 & -128.46 & -17.87 & 0.09 \\
$\Xi_{c}$ & -318.74 & -318.66 & -336.11 & 10.21 & 7.25 \\
$\Sigma_{c}$ & -115.21 & -115.22 & -107.36 & -8.03 & 0.17 \\
$\Xi_{c}^{\prime}$ & -98.95 & -98.95 & -99.02 & -0.58 & 0.65 \\
$\Omega_{c}$ & -94.19 & -94.18 & -97.69 & 2.87 & 0.64 \\
$\Sigma_{c}^{*}$ & -103.57 & -103.72 & -94.23 & -11.03 & 1.55 \\
$\Xi_{c}^{*}$ & -68.87 & -68.84 & -69.27 & -2.00 & 2.43 \\
$\Omega_{c}^{*}$ & -46.66 & -46.57 & -49.63 & 1.21 & 1.84 \\
\hline \hline
\end{tabular}

TABLE VI. Baryon self-energies evaluated with physical meson and baryon masses using the leading order large- $N_{c}$ relations for the axial vector coupling constants (A1). The table collects all contributions of our second scenario with $C_{[\overline{3} 6]}=F_{[\overline{3} 6]}=F_{[\overline{3} \overline{3}]}=0$ and $F_{[66]}=1$ together with $H_{[66]}=-\sqrt{3} C_{[66]}=1.5$.

\begin{tabular}{lccccc}
\hline \hline $\mathrm{B}$ & $\bar{\Sigma}_{B}^{\text {bubble }}$ & $\bar{\Sigma}_{B}^{\text {bubble}-(3+4+5)}$ & $\bar{\Sigma}_{B}^{\text {bubble }-3}$ & $\bar{\Sigma}_{B}^{\text {bubble-4 }}$ & $\bar{\Sigma}_{B}^{\text {bubble-5 }}$ \\
\hline$\Lambda_{c}$ & 0 & 0 & 0 & 0 & 0 \\
$\Xi_{c}$ & 0 & 0 & 0 & 0 & 0 \\
$\Sigma_{c}$ & -276.81 & -276.87 & -237.25 & -44.82 & 5.19 \\
$\Xi_{c}^{\prime}$ & -359.00 & -357.15 & -386.09 & 18.47 & 10.47 \\
$\Omega_{c}$ & -473.89 & -471.62 & -565.31 & 82.41 & 11.29 \\
$\Sigma_{c}^{*}$ & -322.17 & -322.25 & -269.53 & -60.14 & 7.42 \\
$\Xi_{c}^{*}$ & -410.54 & -407.88 & -446.25 & 23.98 & 14.39 \\
$\Omega_{c}^{*}$ & -535.12 & -531.82 & -653.02 & 105.61 & 15.58 \\
\hline \hline
\end{tabular}

two cases. For both scenarios we illustrate with Tables V and VI that our chiral decomposition of the one-loop bubble functions is very well converging.

Consider the first scenario in Table V. The self-energy $\bar{\Sigma}_{B}^{\text {bubble }}$ truncated at the fifth order is reproduced with an uncertainty of at most $0.2 \mathrm{MeV}$. Already with the fourth order term $\bar{\Sigma}_{B}^{\text {bubble-4}}$ the full one-bubble loop function is recovered with an uncertainty of at most $7 \mathrm{MeV}$ only. The contributions from the bubble loop are sizable and can be as large as $320 \mathrm{MeV}$. Thus such contributions will play a decisive role in any chiral extrapolation study of the charmed baryon masses.

We turn to our second scenario in Table VI. Here we do not know the absolute size of the self-energy contributions. The table shows our values at the $a d$-hoc choice $F_{[66]}=1$. For instance at half its value with $F_{[66]}=0.5$ all entries in the table are reduced by a factor of four. Note that according to $[12,13]$ the quark model suggests the value $\left|F_{[66]}\right|=$ $2\left|F_{[36]}\right| / \sqrt{3} \simeq 0.90$. Once a reliable estimate for the axial coupling constant $F_{[66]}$ is available the total contribution of the bubble loop is obtained by adding the values in Table $\mathrm{V}$ with $F_{[66]}^{2}$ times the corresponding values of Table VI. 
In fact such sums may be compared with the values in Table II of the previous work [12], which relies on the heavybaryon mass formulation of $\chi$ PT. From such a comparison we conclude again, that indeed the latter approach does not provide any significant results if truncated at $\mathrm{N}^{2} \mathrm{LO}$ or $\mathrm{N}^{3} \mathrm{LO}$.

Like for the contributions in Table $\mathrm{V}$ we observe a stunning convergence behaviour. The self-energy $\bar{\Sigma}_{B}^{\text {bubble }}$ truncated at the fifth order is reproduced with an uncertainty of about $0.5 \%$. Note that in this scenario the flavor antitriplet baryons do not receive any contributions. This is so since at leading order in the large- $N_{c}$ expansion it holds $F_{[\overline{3} \overline{3}]}=0$.

We conclude that a chiral decomposition of the one-loop contributions formulated in terms of on-shell meson and baryon masses appears well converging also for the charmed baryon masses. Thus, in any realistic application to QCD lattice data, which should be minimally at $\mathrm{N}^{3} \mathrm{LO}$, it is not required to work with loop expressions truncated to some order. Since the fifth order terms are about $10 \mathrm{MeV}$ on average it is well justified to apply the loop functions as they are specified in Chapter III for the finite volume case. This is the strategy followed also in our previous works on the chiral extrapolation of other hadron masses in $[1,2]$. Note that the size of the systematic error in the charmed baryon masses from current QCD lattice ensembles is at least of that size. As we repeatedly emphasized, any significant results from a fit to the lattice data can be expected only if for a given lattice ensemble the set of eight coupled and nonlinear equations is solved that determines the charmed baryon masses.

\section{SUMMARY}

We considered the self-energies for the charmed baryon masses from the chiral Lagrangian with three light flavors at $\mathrm{N}^{3} \mathrm{LO}$. Explicit and renormalization-scale invariant expressions for all ground-state baryons with $J^{P}=\frac{1}{2}^{+}$and $J^{P}=\frac{3}{2}+$ quantum numbers are derived. The results are given in terms of on-shell meson and baryon masses as it is required to obtain significant results that can be applied at physical up, down and strange quark masses. The convergence of the chiral expansion is illustrated at the hand of the one-bubble loop contributions. Given our results significant fits of the low-energy parameters to the data set on charmed baryon masses from the QCD lattice community are feasible. While at leading order in the $1 / N_{c}$ expansion there are 5 unknown parameters, at subleading order we derived the relevance of 21 low-energy parameters.

\section{ACKNOWLEDGMENTS}

Y.H. acknowledges partial support from Suranaree University of Technology, Office of the Higher Education Commission under NRU project of Thailand (SUT-COE: High Energy Physics and Astrophysics) and SUT-CHE-NRU (Grant No. FtR.11/2561).

\section{APPENDIX A}

We provide with Table VII a glossary of physical and technical quantities used throughout this work. Note that our notation is in part context specific. Table VIII summarizes the conventions used for the various low-energy constants.

TABLE VII. Some notations for physical and technical quantities as used in this work.

\begin{tabular}{|c|c|c|c|}
\hline Index & Values & SU(3) multiplet $\rightarrow$ label & Defined in \\
\hline$Q$ & $\pi, K, \bar{K}, \eta$ & {$[8] \rightarrow[8]$} & (1) \\
\hline \multirow[t]{3}{*}{$B, R$} & $\Lambda_{c}, \Xi_{c}$ & {$[\overline{3}] \rightarrow[\overline{3}]$} & (1) \\
\hline & $\Xi_{c}^{\mu}, \Sigma_{c}^{\mu}, \Omega_{c}^{\mu}$ & {$[6] \rightarrow[4]$} & (1) \\
\hline & $\Xi_{c}^{\prime}, \Sigma_{c}, \Omega_{c}$ & {$[6] \rightarrow[6]$} & (1) \\
\hline
\end{tabular}

\begin{tabular}{llc}
\hline \hline Physical quantities & \multicolumn{1}{c}{ Type } & Defined in \\
\hline$m_{Q}$ & Meson mass & PDG \\
$M_{B}, M_{R}$ & Baryon mass & PDG \\
$\Sigma_{B}$ & Baryon self-energy & $(9),(11),(17),(22)$ \\
$G_{B Q}^{(\chi)}$ & Clebsch coefficient & Table I \\
$G_{B Q}^{(S, V)}$ & Clebsch coefficient & Table I \\
$G_{Q R}^{(B)}$ & Clebsch coefficient & Tables III and IV \\
\hline \hline Technical quantities & \multicolumn{1}{c}{ Type } & Defined in \\
\hline$\alpha_{Q R}^{(B)}$ & Subtraction term & $(17),(21),(22),(24)$ \\
$\gamma_{B}^{R}$ & Subtraction term & $(18),(20)$ \\
$\bar{I}_{Q}^{(n)}, \bar{I}_{Q}^{R}$ & Scalar tadpole & $(11),(12),(18)$ \\
$\bar{I}_{Q R}\left(M_{B}\right)$ & Scalar bubble & $(18)$ \\
$a, b$ & Label for multiplets & \\
$\Gamma_{c_{n,[a b]}}$ & $a, b=\overline{3}, 6,4$ & $(25)$, Appendix B \\
$\Gamma_{e_{n,[66]}}$ & Scale dependence & $(25)$, Appendix B \\
\hline \hline & Scale dependence & \\
\hline
\end{tabular}

TABLE VIII. Notation for the low-energy constants as used in this work.

\begin{tabular}{ccc}
\hline \hline LEC & Chiral order & Defined in \\
\hline$M_{[a]}$ & $Q^{0}$ & $(10)$ \\
$F_{[a b]}$ & $Q^{1}$ & $(3)$ \\
$C_{[a b]}$ & $Q^{1}$ & $(3)$ \\
$H_{[a b]}$ & $Q^{1}$ & $(3)$ \\
$g_{n,[a b]}^{(S, V)}, \tilde{g}_{n,[a b]}^{(S, V)}, \bar{g}_{n,[a b]}^{(S, V)}$ & $Q^{2}$ & $(7),(13),(19)$ \\
$h_{n,[a b]}^{(S, V)}, \tilde{h}_{n,[a b]}^{(S, V)}, \bar{h}_{n,[a b]}^{(S, V)}$ & $Q^{2}$ & $(7),(\mathrm{B} 3),(23)$ \\
$b_{n,[a b]}$ & $Q^{2}$ & $(4)$ \\
$d_{n,[a b]}$ & $Q^{2}$ & $(4)$ \\
$c_{n,[a b]}, \tilde{c}_{n,[a b]}$ & $Q^{4}$ & $(8),(14),(16)$ \\
$e_{n,[a b]}, \tilde{e}_{n,[a b]}$ & $Q^{4}$ & $(8),(16)$ \\
\hline \hline
\end{tabular}


The large number of unknown low-energy constants is reduced by sets of sum rules that follow from a systematic $1 / N_{c}$ expansion [10,11]. While at leading order the large- $N_{c}$ operator analysis predicts $36=4+16+16$ sum rules

$$
\begin{aligned}
& H_{[66]}=-\sqrt{3} C_{[66]}=\frac{3}{2} F_{[66]}, \quad C_{[\overline{3} 6]}=\sqrt{3} F_{[\overline{3} 6]}, \quad F_{[\overline{3} \overline{3}]}=0, \\
& b_{n,[66]}=d_{n,[66]}=b_{n,[\overline{3} \overline{3}]} \quad \text { for } n=1,2, \quad b_{1,[\overline{3} 6]}=0, \\
& c_{n,[66]}=e_{n,[66]} \quad \text { for } n=1, \ldots, 5, \\
& c_{n,[\overline{3} 6]}=0 \quad \text { for } n=1,2, \\
& c_{1,[\overline{3} \overline{3}]}=c_{1,[66]}+\frac{1}{2} c_{5,[66]} \quad c_{2,[\overline{3} \overline{3}]}=c_{2,[66]}-\frac{1}{2} c_{5,[66]}, \\
& c_{3,[\overline{3} \overline{3}]}=c_{3,[66]}+2 c_{5,[66]}, \quad c_{4,[\overline{3} \overline{3}]}=c_{4,[66]}-2 c_{5,[66]}, \\
& \bar{g}_{D,[\overline{3} \overline{3}]}^{(S)}=\bar{h}_{2,[66]}^{(S)}-\bar{h}_{4,[66]}^{(S)}-2 \bar{h}_{5,[66]}^{(S)}, \quad \bar{g}_{0,[\overline{3} \overline{3}]}^{(S)}=\frac{1}{2} \bar{h}_{4,[66]}^{(S)}+\bar{h}_{5,[66]}^{(S)}, \\
& \bar{g}_{0,[66]}^{(S)}=\bar{g}_{D,[\overline{3} 6]}^{(S)}=0, \quad \bar{g}_{1,[66]}^{(S)}=\bar{h}_{4,[66]}^{(S)}+\frac{2}{3} \bar{h}_{5,[66]}^{(S)}, \quad \bar{g}_{D,[66]}^{(S)}=\bar{h}_{2,[66]}^{(S)}, \\
& \bar{h}_{0,[66]}^{(S)}=\bar{h}_{1,[66]}^{(S)}=\bar{h}_{3,[66]}^{(S)}=0, \quad \hat{g}_{D,[\overline{3} \overline{3}]}^{(V)}=2 \hat{g}_{1,[\overline{3} \overline{3}]}^{(V)}-\hat{h}_{1,[66]}^{(V)}+\hat{h}_{2,[66]}^{(V)}, \\
& \hat{g}_{0,[\overline{3} \overline{3}]}^{(V)}=-\hat{g}_{1,[\overline{3} \overline{3}]}^{(V)}+\frac{1}{2} \hat{h}_{1,[66]}^{(V)}, \quad \hat{g}_{1,[66]}^{(V)}=\hat{h}_{1,[66]}^{(V)}, \quad \hat{g}_{D,[66]}^{(V)}=\hat{h}_{2,[66]}^{(V)}, \\
& \hat{g}_{0,[66]}^{(V)}=\hat{g}_{D,[\overline{3} 6]}^{(V)}=\hat{h}_{0,[66]}^{(V)}=0, \quad
\end{aligned}
$$

at subleading order there remain $17=3+8+6$ sum rules only

$$
\begin{aligned}
C_{[66]} & =\frac{1}{\sqrt{3}}\left(2 F_{[\overline{3} \overline{3}]}-H_{[66]}\right), \quad F_{[\overline{3} \overline{3}]}=3 F_{[66]}-2 H_{[66]}, \quad F_{[\overline{3} 6]}=\frac{1}{\sqrt{3}} C_{[\overline{3} 6]} \\
b_{1,[66]} & =d_{1,[66]}=b_{1,[\overline{3} \overline{3}]}, \quad b_{1,[\overline{3} 6]}=5 \sqrt{3}\left(b_{2,[66]}-d_{2,[66]}\right), \\
c_{1,[\overline{3} 6]} & =5 \sqrt{3}\left(c_{3,[66]}-e_{3,[66]}\right), \quad c_{2,[66]}=e_{2,[66]}, \\
c_{2,[\overline{3} 6]} & =5 \sqrt{3}\left(c_{4,[66]}-e_{4,[66]}\right), \\
3 c_{1,[\overline{3} \overline{3}]}+c_{2,[\overline{3} \overline{3}]}+c_{4,[\overline{3} \overline{3}]} & =3 c_{1,[66]}+c_{2,[66]}+c_{4,[66]}-c_{5,[66]}, \\
3 e_{1,[66]}+e_{4,[66]}-e_{5,[66]} & =3 c_{1,[66]}+c_{4,[66]}-c_{5,[66]}, \quad \\
\bar{g}_{D,[\overline{3} 6]}^{(S)} & =\frac{1}{\sqrt{3}}\left(\bar{g}_{D,[66]}^{(S)}-\bar{h}_{2,[66]}^{(S)}\right), \quad \bar{g}_{0,[66]}^{(S)}=\bar{h}_{0,[66]}^{(S)}, \quad \bar{h}_{1,[66]}^{(S)}=\bar{h}_{3,[66]}^{(S)}=0, \\
\hat{g}_{D,[\overline{3} 6]}^{(V)} & =\frac{1}{\sqrt{3}}\left(\hat{g}_{D,[66]}^{(V)}-\hat{h}_{2,[66]}^{(V)}\right), \quad \hat{g}_{0,[66]}^{(V)}=\hat{h}_{0,[66]}^{(V)},
\end{aligned}
$$

where we apply the notation

$$
\hat{g}_{n,[a b]}^{(V)}=\frac{2}{M_{[a]}+M_{[b]}} \bar{g}_{n,[a b]}^{(V)}, \quad \hat{h}_{n,[a a]}^{(V)}=\frac{1}{M_{[4]}} \bar{h}_{n,[a a]}^{(V)} .
$$

We close this Appendix with a short summary of the implications from the heavy-quark spin symmetry that arises in the limit of an infinitely heavy charm quark mass $[13,14,19,23]$. The mass parameters $M_{[\overline{3}]}^{1 / 2}, M_{[6]}^{1 / 2}$ and $M_{[6]}^{3 / 2}$ may be expanded in inverse powers of the charm quark mass $M_{c}$. A matching with QCD's properties $[13,14,19,23]$ leads to the scaling properties

$$
M_{[6]}^{3 / 2}-M_{[6]}^{1 / 2} \sim \frac{1}{M_{c}}, \quad M_{[6]}^{1 / 2}-M_{[\overline{3}]}^{1 / 2} \sim M_{c}^{0}
$$


which implies that the two sextet masses are degenerate in this limit. We recall that all sum rules valid at leading order in the $1 / N_{c}$ expansion are compatible with the expectation from the heavy-quark spin symmetry [10]. Note however, that the implications of the large- $N_{c}$ analysis are typically more restrictive, i.e., there are additional constraints arising. The only exception from this is the relation $f_{1}^{(A)}=0$ from
[10] which is requested by the heavy-quark spin symmetry, but not foreseen by the $1 / N_{c}$ expansion. However, the lowenergy constant $f_{1}^{(A)}$ is of no relevance in the current work.

At subleading order in the $1 / N_{c}$ expansion heavy-spin symmetry breaking terms are allowed. For instance $b_{1,[\overline{3} 6]}$ or $c_{n,[\overline{3} 6]}$ need no longer to vanish.

\section{APPENDIX B}

The renormalization scale dependence of the c's and e's as implied is

$$
\mu^{2} \frac{\mathrm{d}}{\mathrm{d} \mu^{2}} c_{i,[a b]}=-\frac{1}{4} \frac{1}{(4 \pi f)^{2}} \Gamma_{c_{i,[a b]}}, \quad \mu^{2} \frac{\mathrm{d}}{\mathrm{d} \mu^{2}} e_{i,[66]}=-\frac{1}{4} \frac{1}{(4 \pi f)^{2}} \Gamma_{e_{i,[66]}},
$$

with $a, b=\overline{3}, 6$ and

$$
\begin{aligned}
& \Gamma_{c_{1,[\overline{3} \overline{3}]}}=\frac{1}{3}\left(10 b_{1,[\overline{3} \overline{3}]}+3 b_{2,[\overline{3} \overline{3}]}\right)-\frac{1}{9}\left(15 \bar{g}_{0,[\overline{3} \overline{3}]}^{(S)}+13 \bar{g}_{D,[\overline{3} \overline{3}]}^{(S)}\right)-\frac{\left.M_{[\overline{[}]}\right]}{36}\left(15 \bar{g}_{0,[\overline{3} \overline{3}]}^{(V)}-11 \bar{g}_{1,[\overline{3} \overline{3}]}^{(V)}+13 \bar{g}_{D,[\overline{3} \overline{3}]}^{(V)}\right) \text {, } \\
& \Gamma_{c_{2,[\overline{3} \overline{3}]}}=\frac{22}{9} b_{1,[\overline{3} \overline{3}]}-\frac{1}{27}\left(33 \bar{g}_{0,[\overline{3} \overline{3}]}^{(S)}-4 \bar{g}_{D,[\overline{3} \overline{3}]}^{(S)}\right)-\frac{M_{[\overline{3}]}}{108}\left(33 \bar{g}_{0,[\overline{3} \overline{3}]}^{(V)}+41 \bar{g}_{1,[\overline{3} \overline{3}]}^{(V)}-4 \bar{g}_{D,[\overline{3} \overline{3}]}^{(V)}\right) \text {, } \\
& \Gamma_{c_{3,[\overline{3} \overline{3}]}}=\frac{22}{9} b_{2,[\overline{3} \overline{3}]}-\frac{26}{9} \bar{g}_{D,[\overline{3} \overline{3}]}^{(S)}+\frac{M_{[\overline{3}]}}{36}\left(52 \bar{g}_{1,[\overline{3} \overline{3}]}^{(V)}-26 \bar{g}_{D,[\overline{3} \overline{3}]}^{(V)}\right), \\
& \Gamma_{c_{4,[\overline{3} \overline{3}]}}=\frac{1}{3} b_{2,[\overline{3} \overline{3}]}+\bar{g}_{D,[\overline{3} \overline{3}]}^{(S)}+\frac{M_{[\overline{3}]}}{4}\left(\bar{g}_{D,[\overline{3} \overline{3}]}^{(V)}-2 \bar{g}_{1,[\overline{3} \overline{3}]}^{(V)}\right) \text {, } \\
& \Gamma_{c_{1, \overline{3} 6]}}=\frac{22}{9} b_{1,[\overline{3} 6]}-\frac{26}{9} \bar{g}_{D,[\overline{3} 6]}^{(S)}-\frac{13}{36}\left(M_{[\overline{3}]}+M_{[6]}\right) \bar{g}_{D,[\overline{[} 6]}^{(V)}, \\
& \Gamma_{c_{2, \overline{3} 6]}}=\frac{1}{3} b_{1,[\overline{3} 6]}+\bar{g}_{D,[\overline{3} 6]}^{(S)}+\frac{1}{8}\left(M_{[\overline{3}]}+M_{[6]}\right)_{D,[\overline{3} 6]}^{(V)}, \\
& \Gamma_{c_{1,[6]}}=\frac{1}{3}\left(10 b_{1,[66]}+3 b_{2,[66]}\right)-\frac{1}{9}\left(15 \bar{g}_{0,[66]}^{(S)}+2 \bar{g}_{1,[66]}^{(S)}+13 \bar{g}_{D,[66]}^{(S)}\right)-\frac{M_{[6]}}{36}\left(15 \bar{g}_{0,[66]}^{(V)}+2 \bar{g}_{1,[66]}^{(V)}+13 \bar{g}_{D,[66]}^{(V)}\right), \\
& \Gamma_{c_{2,[6]}}=\frac{22}{9} b_{1,[66]}-\frac{1}{27}\left(33 \bar{g}_{0,[66]}^{(S)}-2 \bar{g}_{1,[66]}^{(S)}-4 \bar{g}_{D,[66]}^{(S)}\right)-\frac{M_{[6]}}{108}\left(33 \bar{g}_{0,[66]}^{(V)}-2 \bar{g}_{1,[66]}^{(V)}-4 \bar{g}_{D,[66]}^{(V)}\right), \\
& \Gamma_{c_{3,[6]}}=\frac{22}{9} b_{2,[66]}-\frac{2}{9}\left(2 \bar{g}_{1,[66]}^{(S)}+13 \bar{g}_{D,[66]}^{(S)}\right)-\frac{M_{[6]}}{18}\left(2 \bar{g}_{1,[66]}^{(V)}+13 \bar{g}_{D,[66]}^{(V)}\right) \text {, } \\
& \Gamma_{c_{4,[66]}}=\frac{1}{3} b_{2,[66]}+\frac{1}{3}\left(\bar{g}_{1,[66]}^{(S)}+3 \bar{g}_{D,[66]}^{(S)}\right)+\frac{M_{[6]}}{12}\left(\bar{g}_{1,[66]}^{(V)}+3 \bar{g}_{D,[66]}^{(V)}\right) \text {, } \\
& \Gamma_{c_{5,[66]}}=-\frac{1}{3} \bar{g}_{1,[66]}^{(S)}-\frac{M_{[6]}}{12} \bar{g}_{1,[66]}^{(V)} \text {, } \\
& \Gamma_{e_{1,[6]}}=\frac{1}{3}\left(10 d_{1,[66]}+3 d_{2,[66]}\right)-\frac{1}{9}\left(15 \tilde{h}_{0,[66]}^{(S)}+2 \tilde{h}_{1,[66]}^{(S)}+13 \tilde{h}_{2,[66]}^{(S)}\right)-\frac{M_{[4]}}{36}\left(15 \tilde{h}_{0,[66]}^{(V)}+2 \tilde{h}_{1,[66]}^{(V)}+13 \tilde{h}_{2,[66]}^{(V)}\right), \\
& \Gamma_{e_{2,[6]]}}=\frac{22}{9} d_{1,[66]}-\frac{1}{27}\left(33 \tilde{h}_{0,[66]}^{(S)}-2 \tilde{h}_{1,[66]}^{(S)}-4 \tilde{h}_{2,[66]}^{(S)}\right)-\frac{M_{[4]}}{108}\left(33 \tilde{h}_{0,[66]}^{(V)}-2 \tilde{h}_{1,[66]}^{(V)}-4 \tilde{h}_{2,[66]}^{(V)}\right), \\
& \Gamma_{e_{3,[6]}}=\frac{22}{9} d_{2,[66]}-\frac{2}{9}\left(2 \tilde{h}_{1,[66]}^{(S)}+13 \tilde{h}_{2,[66]}^{(S)}\right)-\frac{M_{[4]}}{18}\left(2 \tilde{h}_{1,[66]}^{(V)}+13 \tilde{h}_{2,[66]}^{(V)}\right), \\
& \Gamma_{e_{4,[6]}}=\frac{1}{3} d_{2,[66]}+\frac{1}{3}\left(\tilde{h}_{1,[66]}^{(S)}+3 \tilde{h}_{2,[66]}^{(S)}\right)+\frac{M_{[4]}}{12}\left(\tilde{h}_{1,[66]}^{(V)}+3 \tilde{h}_{2,[66]}^{(V)}\right), \\
& \Gamma_{e_{5,[6]}}=-\frac{1}{3} \tilde{h}_{1,[66]}^{(S)}-\frac{M_{[4]}}{12} \tilde{h}_{1,[66]}^{(V)},
\end{aligned}
$$

and 


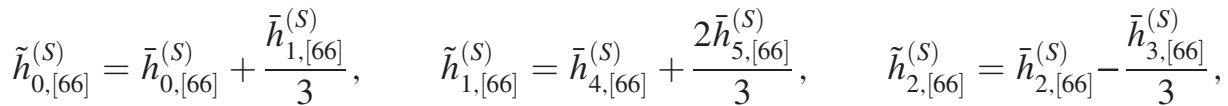

$$
\begin{aligned}
& \tilde{h}_{0,[66]}^{(V)}=\bar{h}_{0,[66]}^{(V)}-\frac{\bar{h}_{1,[66]}^{(S)}}{3 M_{[4]}}, \quad \tilde{h}_{1,[66]}^{(V)}=\bar{h}_{1,[66]}^{(V)}-\frac{2 \bar{h}_{5,[66]}^{(S)}}{3 M_{[4]}}, \quad \tilde{h}_{2,[66]}^{(V)}=\bar{h}_{2,[66]}^{(V)}+\frac{\bar{h}_{3,[66]}^{(S)}}{3 M_{[4]}} .
\end{aligned}
$$

[1] M. F. M. Lutz, Y. Heo, and X.-Y. Guo, Nucl. Phys. A977, 146 (2018).

[2] X.-Y. Guo, Y. Heo, and M. F. M. Lutz, Phys. Rev. D 98, 014510 (2018).

[3] R. Bavontaweepanya, X.-Y. Guo, and M. F. M. Lutz, Phys. Rev. D 98, 056005 (2018).

[4] L. Liu, H.-W. Lin, K. Orginos, and A. Walker-Loud, Phys. Rev. D 81, 094505 (2010).

[5] G. Bali, S. Collins, and P. Perez-Rubio, J. Phys. Conf. Ser. 426, 012017 (2013).

[6] R. A. Briceno, H.-W. Lin, and D. R. Bolton, Phys. Rev. D 86, 094504 (2012).

[7] C. Alexandrou, J. Carbonell, D. Christaras, V. Drach, M. Gravina, and M. Papinutto, Phys. Rev. D 86, 114501 (2012).

[8] Y. Namekawa et al. (PACS-CS Collaboration), Phys. Rev. D 87, 094512 (2013).

[9] P. Perez Rubio, Proc. Sci., ConfinementX2012 (2012) 141 [arXiv:1302.5774].

[10] M. F. M. Lutz, D. Samart, and Y. Yan, Phys. Rev. D 90, 056006 (2014).

[11] Y. Heo and M. F. M. Lutz, Phys. Rev. D 97, 094004 (2018).

[12] N. Jiang, X.-L. Chen, and S.-L. Zhu, Phys. Rev. D 90, 074011 (2014).
[13] T.-M. Yan, H.-Y. Cheng, C.-Y. Cheung, G.-L. Lin, Y. Lin, and H.-L. Yu, Phys. Rev. D 46, 1148 (1992).

[14] P. L. Cho, Phys. Lett. B 285, 145 (1992).

[15] C. Terschlsen, S. Leupold, and M. F. M. Lutz, Eur. Phys. J. A 48, 190 (2012).

[16] X.-K. Guo, Z.-H. Guo, J. A. Oller, and J. J. Sanz-Cillero, J. High Energy Phys. 06 (2015) 175.

[17] S. Okubo, Phys. Lett. 5, 165 (1963).

[18] M. F. M. Lutz and E. Kolomeitsev, Nucl. Phys. A730, 110 (2004).

[19] E. E. Jenkins, Phys. Rev. D 54, 4515 (1996).

[20] A. Semke and M. F. M. Lutz, Nucl. Phys. A778, 153 (2006).

[21] M. F. M. Lutz, R. Bavontaweepanya, C. Kobdaj, and K. Schwarz, Phys. Rev. D 90, 054505 (2014).

[22] C. Patrignani et al. (Particle Data Group), Chin. Phys. C 40, 100001 (2016).

[23] H. Georgi, Nucl. Phys. B348, 293 (1991).

Correction: The given name of the second author contained an error and has been fixed. 